\title{
3D Seiberg-like dualities and M2 branes
}

\author{
Antonio Amariti, ${ }^{a, b}$ Davide Forcella, ${ }^{c, d}$ Luciano Girardello ${ }^{a, b}$ and Alberto Mariotti ${ }^{e, f}$ \\ ${ }^{a}$ Dipartimento di Fisica, Università di Milano Bicocca, \\ Milano, Italy \\ ${ }^{b}$ INFN, Sezione di Milano-Bicocca, \\ Piazza della Scienza 3, I-20126 Milano, Italy \\ ${ }^{c}$ Laboratoire de Physique Théorique de l'École Normale Supérieure, \\ Paris, France \\ ${ }^{d}$ CNRS UMR 8549, \\ 24 Rue Lhomond, Paris 75005, France \\ e Theoretische Natuurkunde, Vrije Universiteit Brussel, \\ Brussels, Belgium \\ ${ }^{f}$ The International Solvay Institutes, \\ Pleinlaan 2, B-1050 Brussels, Belgium \\ E-mail: antonio.amariti@mib.infn.it, forcella@lpt.ens.fr, \\ luciano.girardello@mib.infn.it, alberto.mariotti@vub.ac.be
}

Abstract: We investigate features of duality in three dimensional $\mathcal{N}=2$ Chern-Simons matter theories conjectured to describe M2 branes at toric Calabi Yau four-fold singularities. For 3D theories with non-chiral 4D parents we propone a Seiberg-like duality which turns out to be a toric duality. For theories with chiral 4D parents we discuss the conditions under which that Seiberg-like duality leads to toric duality. We comment on such duality in $3 \mathrm{D}$ theories without $4 \mathrm{D}$ parents.

Keywords: Supersymmetry and Duality, Duality in Gauge Field Theories, Chern-Simons Theories, Brane Dynamics in Gauge Theories 


\section{Contents}

1 Introduction 1

2 M2 branes and $\mathcal{N}=2$ Chern Simons theories 3

2.1 An algorithm to compute $\mathcal{M}_{4}$

3 Non-chiral theories $\quad 6$

3.1 Seiberg-like duality 8

$3.2 \widetilde{L^{121}}\left\{k_{1}, k_{2}, k_{3}\right\} \quad 9$

$\begin{array}{ll}3.3 \widetilde{L^{222}}\left\{k_{i}\right\} & 11\end{array}$

3.4 The general $\widetilde{L^{a b a}}\left\{k_{i}\right\} \quad 13$

4 Chiral theories $\quad 16$

$\begin{array}{lll}4.1 & \widetilde{\mathbb{F}_{0}}\left\{k_{i}\right\} & 17\end{array}$

$\begin{array}{lll}4.2 & \widetilde{d P}_{1\left\{k_{i}\right\}} & 20\end{array}$

$4.3 \widetilde{d P_{2}}\left\{k_{i}\right\} \quad 21$

$4.4 \widetilde{d P_{3}\left\{k_{i}\right\}} \quad 22$

$4.5 \widetilde{Y^{32}}\left\{k_{i}\right\} \quad 24$

5 Dualities for CS theories without 4d parents 26

$\begin{array}{ll}5.1 \text { Example } 26 & 26\end{array}$

$\begin{array}{llr}6 & \text { Conclusion } & 27\end{array}$

$\begin{array}{lr}\text { A Parity anomaly } & 28\end{array}$

\section{Introduction}

Different descriptions of the same physical phenomenon usually provide a better understanding of the phenomenon itself. AdS/CFT correspondence and Seiberg duality are two famous examples. In the $\mathrm{AdS}_{5} / \mathrm{CFT}_{4}$ case it happens that to a single geometry correspond different UV field theory descriptions. This phenomenon was called Toric Duality in [1], analyzed in $[2,3]$ and identified as a Seiberg duality in $[4,5]$. Due to the difficulties to understand the field theory living on $\mathrm{M} 2$ branes the $\mathrm{AdS}_{4} / \mathrm{CFT}_{3}$ correspondence was less mastered. An important step in this direction has been the realization of the importance of Chern-Simons interactions [6] and the subsequent construction of $\mathcal{N}=8$ Chern-Simons matter field theories in [7-10, 10-14]. A breakthrough toward the explicit realization of the $\mathrm{AdS}_{4} / \mathrm{CFT}_{3}$ correspondence was done in [15] where the authors propose $\mathrm{U}(N)_{k} \times \mathrm{U}(N)_{-k}$ 
CS matter theories, with $\mathcal{N}=6$ supersymmetry, to be the low energy theories of $N$ M2branes at the $\mathbb{C}^{4} / \mathbb{Z}_{k}$ singularities. Afterward, this construction has been extended to many others CS matter theories with a lower amount of supersymmetries [16-25, 27-29].

A large and interesting, but still very peculiar, class of $\mathrm{AdS}_{4} / \mathrm{CFT}_{3}$ pairs is realized by M2 branes at Calabi Yau four-fold toric singularities [20-22, 28-31]. The low energy theories are proposed to be a special kind of $\mathcal{N}=2$ Chern-Simons matter theories. It was soon realized [22] that, as in the $\mathrm{AdS}_{5} / \mathrm{CFT}_{4}$ case, different $\mathcal{N}=2$ Chern-Simons matter theories can be associated with the same Calabi Yau fourfold geometry. The phenomenon of toric duality reappears in the $\mathrm{AdS}_{4} / \mathrm{CFT}_{3}$ correspondence, but in a much more general context. For example, contrary to the four dimensional case, in three dimensional gauge theories one find models with different numbers of gauge group factors to describe the same IR physics (for example some of the mirror symmetry pairs $[32,33]$ ). In the literature some very specific pairs of dual field theories were constructed. A step was done in [34, 35] where a sort of Seiberg like duality for three dimensional Chern-Simons matter theory was proposed. ${ }^{1}$ In the context of M2 branes at singularities, we can divide the set of dualities into the ones that change and in the ones that do not change the number of gauge group factors. In this paper we will call the second type of duality Seiberg-like toric duality.

$\mathcal{N}=2$ Chern-Simons matter theories for M2 branes at singularities are typically described by a quiver with an assignment of Chern-Simons levels $k_{i}$ and a superpotential in a way similar to the gauge theories for D3 branes at Calabi Yau three-fold singularities. Indeed a class of $\mathcal{N}=2$ three dimensional theories can be simply obtained from four dimensional $\mathcal{N}=1$ quivers with superpotential in the following way: rewrite the theory in three dimensions, change the $\mathrm{SU}(N)$ gauge factors to $\mathrm{U}(N)$ factors, disregard the super Yang-Mills actions and add a super Chern-Simons term for every factors. We will say that these three dimensional theories have a four dimensional parent. Viceversa we will call theories without four dimensional parents the three dimensional theories that cannot be obtained in the way just explained [29].

In this paper we investigate Seiberg-like toric dualities for $(2+1)$ dimensional $\mathcal{N}=2$ Chern-Simons matter theories associated with M2 branes at Calabi Yau four-fold toric singularities. Using a generalization of the forward algorithm for D3 branes [28] we analyze a particular branch of the moduli space that is supposed to reproduce the transverse four-fold Calabi Yau singularity $\mathcal{M}_{4}$. N M2-branes at a singularity are mutually BPS and their moduli space will be a $N$-times symmetric product of the moduli space for a single brane. Moreover on a generic point of $\mathcal{M}_{4}$ the gauge group is completely broken and we do not expect quantum corrections. For these reasons and for simplicity we restrict to the classical abelian moduli space.

In the following we will check that different Chern-Simons matter theories describe the same IR dynamics of $N M 2$ branes on a particular four dimensional CY cone by comparing the $\mathcal{M}_{4}$ branch of the moduli space. Our computation is classical and restricted to a particular branch of the abelian theory. It will be very interesting to compute the full moduli space for the non abelian theories, but we argue that the match of the $\mathcal{M}_{4}$ branch

\footnotetext{
${ }^{1}$ Seiberg duality for 3D gauge theories were previously studied in [36-38].
} 
of the moduli space is already a good check of the toric duality.

For theories with four dimensional parents one could try to simply extend the four dimensional Seiberg duality to the three dimensional case. In fact, three dimensional theories share the same Master Spaces [39, 40] of their four dimensional parents. In the map between four and three dimensions a direction of the Master Space become a direction of the physical Calabi Yau four-fold. Unfortunately, it turns out that an arbitrary assignment of $2+1$ dimensional Chern-Simons levels does not in general commute with $3+1$ dimensional Seiberg duality [22]. In fact it was shown in [41] that the Master Space for four dimensional Seiberg dual theories are not in general isomorphic. Actually it seems that three dimensional CS theories with chiral four dimensional parents do not admit a simple generalization of the three dimensional SQCD Seiberg duality as it happens in the four dimensional case.

Here, we first analyze non chiral three dimensional CS theories with $(3+1) d$ parents. Using a type $I I B$ brane realization, we propose a Seiberg like duality, with a precise prescription for the transformation of the CS levels and the gauge groups factors. We then check that this proposed Seiberg like duality is indeed a toric duality, namely that the two dual theories are associated with M2 branes probing the same Calabi Yau four-fold singularity.

We try to simply extend to chiral CS theories with $(3+1)$ d parents the rules that we have found for the non chiral theories. For chiral four dimensional theories the Master Space is not isomorphic among Seiberg dual phases [41]. This fact presumably puts constraints on the duality transformations for the three dimensional case. We find indeed difficulties for a straightforward realization of Seiberg like toric dualities for $2+1$ dimensional Chern-Simons matter theories with four dimensional chiral parents.

However, by analyzing several examples, we find a rule for the assignments of the CS levels such that toric duality still holds among Seiberg like dual phases.

We finally give some examples of Chern-Simons theories without four dimensional parents. In this case there is no immediate insight from the four dimensions, but we show that the duality proposed for the chiral theories works also for theories without a $3+1$ parents.

Our analysis is a first step to the study of Seiberg-like toric dualities in the context of M2 branes. We tried to use the intuition from the non-chiral case and to leave as arbitrary as possible the values of the Chern-Simons levels. It is reasonable that more general transformation rules exist. Moreover it would be nice to investigate more general families of toric dualities, like the ones changing the number of gauge group factors and the large limit for Chern-Simons levels. We leave these topics for future investigations.

As we were finishing this paper, we were informed of $[59,60]$ which discuss related topics.

\section{M2 branes and $\mathcal{N}=2$ Chern Simons theories}

As discussed in the introduction, supersymmetric Chern Simons theories coupled to matter fields are good candidates to describe the low energy dynamics of M2 branes [6, 15]. In this paper we are interested in M2 branes at Calabi Yau four fold toric conical singularities. The authors of $[20,21]$ proposed that the field theories living on these M2 branes are 
$(2+1)$ dimensional $\mathcal{N}=2$ Chern Simons theories with gauge group $\prod_{i=1}^{G} U_{i}(N)$ with bifundamental and adjoint matter fields. The Lagrangian in $\mathcal{N}=2$ superspace notation is:

$$
\operatorname{Tr}\left(-i \sum_{a} k_{a} \int_{0}^{1} d t V_{a} \bar{D}^{\alpha}\left(e^{t V_{a}} D_{\alpha} e^{-t V_{a}}\right)-\int d^{4} \theta \sum_{X_{a b}} X_{a b}^{\dagger} e^{-V_{a}} X_{a b} e^{V_{b}}+\int d^{2} \theta W\left(X_{a b}\right)+c . c .\right)
$$

where $V_{a}$ are the vector superfields and $X_{a b}$ are bifundamental chiral superfields. The superpotential $W\left(X_{a b}\right)$ satisfies the toricity conditions: every field appears just two times: one time with plus sign and the other time with minus sign. Since these theories are conjectured to be dual to $M$ theory on $A d S_{4} \times S E_{7}$, where $S E_{7}$ is a seven dimensional Sasaki Einstein manifold, the moduli space of these theories must contain a branch isomorphic to the four-fold Calabi Yau real cone over $S E_{7}: \mathcal{M}_{4}=C\left(S E_{7}\right)$. To study the moduli space we need to find the vanishing conditions for the scalar potential. The scalar potential is:

$$
\operatorname{Tr}\left(-4 \sum_{a} k_{a} \sigma_{a} D_{a}+\sum_{a} D_{a} \mu_{a}(X)-\sum_{X_{a b}}\left|\sigma_{a} X_{a b}-X_{a b} \sigma_{b}\right|^{2}-\sum_{X_{a b}}\left|\partial_{X_{a b}} W\right|^{2}\right)
$$

where $\mu_{a}(X)=\sum_{b} X_{a b} X_{a b}^{\dagger}-\sum_{c} X_{c a}^{\dagger} X_{c a}+\left[X_{a a}, X_{a a}^{\dagger}\right], \sigma_{a}$ and $D_{a}$ are scalar components of the vector superfield $V_{a}$, and with abuse of notation $X_{a b}$ is the lowest scalar component of the chiral superfield $X_{a b}$. The moduli space is the zero locus of the scalar potential and it is given by the equations:

$$
\partial_{X_{a b}} W=0, \quad \sigma_{a} X_{a b}-X_{a b} \sigma_{b}=0, \quad \mu_{a}(X)=4 k_{a} \sigma_{a} .
$$

In $[20,21]$ it was shown that if

$$
\sum_{a} k_{a}=0
$$

then the moduli space contains a branch isomorphic to a four-fold Calabi Yau singularity. This branch is interpreted as the space transverse to the M2 branes. Let us start with the abelian case in which the gauge group is $\mathrm{U}(1)^{G}$. We are interested in the branch in which all the bifundamental fields are generically different from zero. In this case the solution to the first equation in (2.2) gives the irreducible component of the master space ${ }^{\operatorname{Irr}} \mathcal{F}^{b}[39,40]$. The second equation in (2.2) imposes $\sigma_{a_{1}}=\cdots=\sigma_{a_{G}}=\sigma$. The last equation in (2.2) are $G$ equations; the sum of all the equations gives zero and there are just $G-1$ linearly independent equations. The remaining $G-1$ equations can be divided in one along the direction of the Chern Simons levels, and $G-2$ perpendicular to the direction of the Chern Simons levels. The first equation fixes the value of the field $\sigma$ while the other $G-2$ equations looks like $\mu_{i}(X)=0$ and can be imposed, together with their corresponding $\mathrm{U}(1)$ gauge transformations, modding $\operatorname{Irr}^{b}$ by the complexified gauge group action $\left(\mathbb{C}^{*}\right)^{G-2}$. The equation fixing the field $\sigma$ leaves a $\mathbb{Z}_{k}$ action with $\operatorname{gcd}\left(\left\{k_{\alpha}\right\}\right)=k$ by which we need to quotient to obtain the moduli space. In the following we will take $\operatorname{gcd}\left(\left\{k_{\alpha}\right\}\right)=1$. Summarizing, the branch of the moduli space we just analyzed is:

$$
\mathcal{M}_{4}=\operatorname{Irr}^{b} \mathcal{F}^{b}
$$


where $H$ is the $\left(\mathbb{C}^{*}\right)^{G-2}$ kernel of

$$
C=\left(\begin{array}{cccccc}
1 & 1 & 1 & 1 & 1 & 1 \\
k_{1} & k_{2} & \ldots & \ldots & k_{G-1} & k_{G}
\end{array}\right)
$$

$\operatorname{Irr}^{b}$ is a $G+2$ dimensional toric Calabi Yau cone [39, 40] and the vectors of charges in $H$ are traceless by construction; it implies that $\mathcal{M}_{4}$ is a four dimensional Calabi Yau cone and it is understood as the transverse space to the $M 2$ branes. Following the same procedure in the non abelian case it is possible to see that the moduli space contains the $N$-times symmetric product of $\mathcal{M}_{4}$, and it is interpreted as the the transverse space to a set of $N$ BPS M2 branes.

It is quite generic that a specific Calabi Yau four-fold is a branch of the moduli space of apparently completely different $\mathcal{N}=2$ Chern-Simons theories. This fact it is called toric duality. We want to systematically study $\mathcal{M}_{4}$ for some set of Chern Simons theories and see if it is possible to find examples of toric dual pairs. To do this we will use the algorithm proposed in [28].

\subsection{An algorithm to compute $\mathcal{M}_{4}$}

Let us review the algorithm proposed in [28] to compute $\mathcal{M}_{4}$. We consider an $\mathcal{N}=2$ Chern-Simons theory described in the previous section with gauge group $\mathrm{U}(1)^{G}$, and with the following constraints on the Chern-Simons levels:

$$
\sum k_{a}=0 \quad \operatorname{gcd}\left(\left\{k_{a}\right\}\right)=1
$$

To compute $\mathcal{M}_{4}$ we need three matrices: the incidence matrix $d$, the perfect matching matrix $P$, and the Chern-Simons levels matrix $C . d$ contains the charges of the chiral fields under the gauge group $\mathrm{U}(1)$ factors of the theory, and can be easily obtained from the quiver. $P$ is a map between the gauge linear sigma model variables and the chiral fields in the Chern-Simons theory. It can be obtained from the superpotential of the theory and we refer the reader to $[28,29]$ for explanations. Summarizing, the determination of the field theory contains the three matrices $d, P, C$. They are defined respectively by the gauge group representations of the chiral fields, the chiral fields interactions and the Chern Simons levels. Once we get these three matrices we can obtain the toric diagram of $\mathcal{M}_{4}$. From $P$ and $d$ we compute the matrix $Q$. It is the matrix of charges of the gauge linear sigma model variables under the $\mathrm{U}(1)^{G}$ gauge group: $d=Q \cdot P^{T}$. From $Q$ and $C$ we construct the charge matrix $Q_{D}=\operatorname{ker}(C) \cdot Q$. We denote with $K \equiv \operatorname{ker}(C)$. From $P$ we get the charge matrix $Q_{F}$ : $Q_{F}=\operatorname{ker}\left(P^{T}\right)$. Once we have $Q_{D}$ and $Q_{F}$ we combine them in the total charge matrix $Q_{t}$ :

$$
Q_{t}=\left(\begin{array}{c}
Q_{D} \\
Q_{F}
\end{array}\right)
$$

The toric diagram of $\mathcal{M}_{4}$ is given by the kernel of $Q_{t}$ :

$$
G_{t}=\left(\operatorname{ker}^{*}\left(Q_{t}\right)\right)^{T}
$$




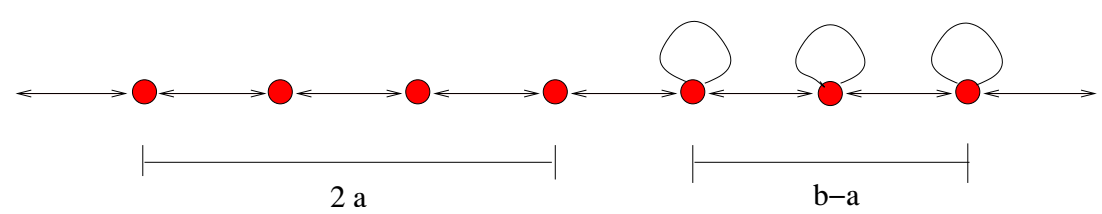

Figure 1. The quiver for the generic $\widetilde{L^{a b a}}\left\{k_{i}\right\}$.

where the columns of $G_{t}$ are the vectors defining the toric diagram of $\mathcal{M}_{4}$. Note that, as pointed out in [28], we have to find the integer kernel, that we denote ker*, and not the nullspace of the charge matrix. Each row of $G_{t}$ is reduced to a basis over the integer for every choice of the CS levels. We will see this algorithm at work in the following sections.

\section{$3 \quad$ Non-chiral theories}

We consider non-chiral 3D $\mathcal{N}=2 \mathrm{CS}$ matter quiver gauge theories which are the three dimensional analog of the four dimensional $L^{a b a}$ theories [42-44]. and will be denoted as $\widetilde{L^{a b a}}\left\{k_{i}\right\}$. We say that a theory is non chiral if for every pair of gauge group factors $\mathrm{U}(N)_{i}$ and $\mathrm{U}(N)_{i+1}$ the number of bifundamental fields in the representation $\left(N_{i}, \bar{N}_{i+1}\right)$ is the same as the number of bifundamental fields in the conjugate representation $\left(\bar{N}_{i}, N_{i+1}\right)$. Otherwise the theory is chiral. The quiver for the $\widetilde{L^{a b a}}\left\{k_{i}\right\}$ is in figure 1, with gauge groups $\prod_{i} \mathrm{U}(N)_{k_{i}}$. We label the nodes from left to right. The action is

$$
\begin{aligned}
S= & \sum_{i} S_{\mathrm{CS}}\left(k_{i}, V_{i}\right) \\
& +\int d^{4} \theta \operatorname{Tr} \sum_{i}\left(e^{-V_{i}} Q_{i, i+1}^{\dagger} e^{V_{i+1}} Q_{i, i+1}+e^{V_{i}} Q_{i+1, i} e^{-V_{i+1}} Q_{i+1, i}^{\dagger}\right)+\sum_{j} X_{j, j}^{\dagger} e^{-2 V_{j}} X_{j, j} \\
& +\int d^{2} \theta \sum_{l}(-1)^{l} \operatorname{Tr} Q_{l-1, l} Q_{l, l+1} Q_{l+1, l} Q_{l, l-1}+\sum_{j} \operatorname{Tr} Q_{j-1, j} X_{j, j} Q_{j, j-1}-Q_{j+1, j} X_{j, j} Q_{j, j+1}
\end{aligned}
$$

where

$$
i=1, \ldots a+b, \quad j=2 a+1 \ldots a+b, \quad l=1 \ldots 2 a,
$$

and $S_{\mathrm{CS}}\left(k_{i}, V_{i}\right)$ is the first term in (2.1).

\section{Brane construction}

3D gauge theories can be engineered in type IIB string theory as $D 3$ branes suspended among five branes [45]. For 3d CS theories the setup includes $(p, q) 5$ branes [46, 47]. Here we construct $\mathcal{N}=2$ three dimensional $\widetilde{L^{a b a}}{ }_{\left\{k_{i}\right\}}$ CS theories, in analogy with the $4 \mathrm{D}$ construction [48].

As an example we show in figure 2 the realization of the $\widetilde{L^{121}}\left\{k_{1}, k_{2}, k_{3}\right\}$ theory. The generalization to the $\widetilde{L^{a b a}}\left\{k_{i}\right\}$ is straightforward. We have the brane content resumed in table 1 . The NS branes and the corresponding D5 branes get deformed in $\left(1, p_{i}\right)$ five branes at angles $\tan \theta_{i} \simeq p_{i}$, obtaining 


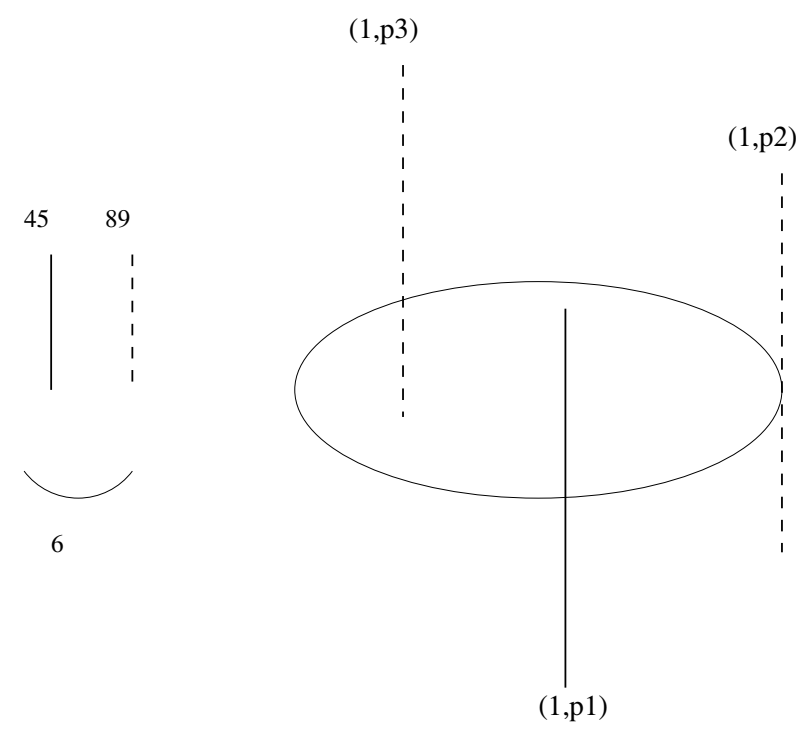

Figure 2. Brane construction for $\widetilde{L^{121}}\left\{k_{1}, k_{2}, k_{3}\right\}$.

\begin{tabular}{|c|c|c|}
\hline$\#$ & brane & directions \\
\hline $\mathrm{N}$ & $\mathrm{D} 3$ & 0126 \\
\hline 1 & $\mathrm{NS}_{1}$ & 012345 \\
\hline 1 & $\mathrm{NS}_{2}$ & 012389 \\
\hline 1 & $\mathrm{NS}_{3}$ & 012389 \\
\hline$p_{1}$ & $\mathrm{D} 5_{1}$ & 012457 \\
\hline$p_{2}$ & $\mathrm{D} 5_{2}$ & 012789 \\
\hline$p_{3}$ & $\mathrm{D} 5_{3}$ & 012789 \\
\hline
\end{tabular}

Table 1. Brane content for the $\widetilde{L^{121}}{ }_{\left\{k_{1}, k_{2}, k_{3}\right\}}$ theory.

- N D3 brane along 0126

- $\left(1, p_{1}\right)$ brane along $012[3,7]_{\theta_{1}} 45$

- $\left(1, p_{2}\right)$ brane along $012[3,7]_{\theta_{2}} 89$

- $\left(1, p_{3}\right)$ brane along $012[3,7]_{\theta_{3}} 89$

Since the $\left(1, p_{2}\right)$ and the $\left(1, p_{3}\right)$ branes are parallel in the 89 direction there is a massless adjoint field on the node 2 . This brane system gives the three dimensional $\widetilde{L^{121}}\left\{k_{1}, k_{2}, k_{3}\right\}$ CS theory. The Chern-Simons levels are associated with the relative angle of the branes in the $[3,7]$ directions, i.e.

$$
k_{i}=p_{i}-p_{i+1} \quad i=1, \ldots, a+b
$$

automatically satisfying (2.3). The gauge groups are all $\mathrm{U}(N)$. Similar brane configurations have been studied in [23, 25-27] for $\mathcal{N}=3$ and/or non toric theories. 


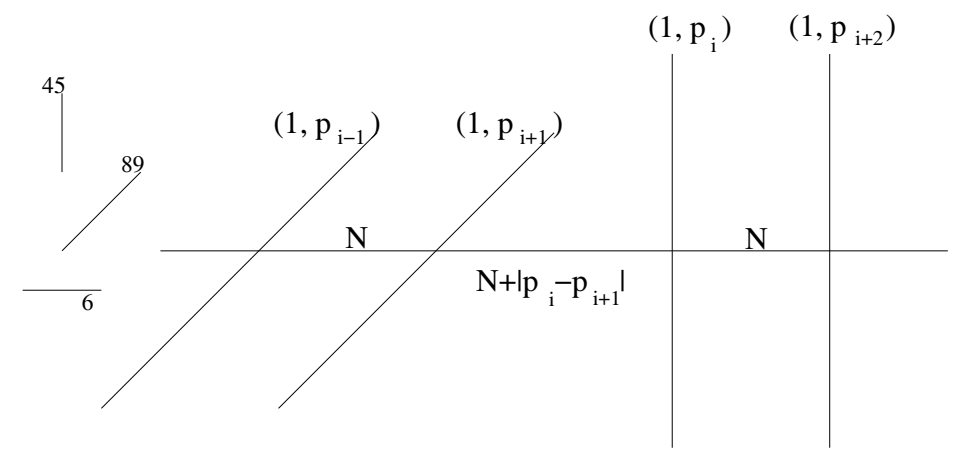

Figure 3. Configuration after exchanging the position of the $\left(1, p_{i}\right)$ and $\left(1, p_{i+1}\right)$ branes. The movement implies that $\left|p_{i}-p_{i+1}\right| D 3$ are created in the middle interval. The rank of the dualized group is $N+\left|p_{i}-p_{i+1}\right|=N+\left|k_{i}\right|$. The CS levels change as $\left(p_{i-1}-p_{i}, p_{i}-p_{i+1}, p_{i+1}-p_{i+2}\right) \rightarrow$ $\left(p_{i-1}-p_{i+1}, p_{i+1}-p_{i}, p_{i}-p_{i+2}\right)$.

\subsection{Seiberg-like duality}

As in the four dimensional case [49], we argue that electric magnetic duality corresponds to the exchange of two orthogonal $\left(1, p_{i}\right)$ and $\left(1, p_{i+1}\right)$ branes. Our starting configuration has only regular branes. When we exchange the five-branes, $\left|p_{i}-p_{i+1}\right|=\left|k_{i}\right|$ D3 branes are created [46]. Observe that, since the $p_{i}$ and $p_{i+1}$ of the dualized gauge group are exchanged, this gives non trivial transformations also for the CS level of the neighbor nodes.

From the brane picture (see figure 3) we obtain the rules for a Seiberg like duality on a node without adjoint fields in the $\widetilde{L^{a b a}}\left\{k_{i}\right\}$ quiver gauge theories. Duality on the $i$-th node gives

$$
\begin{aligned}
\mathrm{U}(N)_{k_{i}} & \rightarrow \mathrm{U}\left(N+\left|k_{i}\right|\right)_{-k_{i}} \\
\mathrm{U}(N)_{k_{i-1}} & \rightarrow \mathrm{U}(N)_{k_{i-1}+k_{i}} \\
\mathrm{U}(N)_{k_{i+1}} & \rightarrow \mathrm{U}(N)_{k_{i+1}+k_{i}}
\end{aligned}
$$

and the field content and the superpotential changes as in 4D Seiberg duality.

The duality that we derived from the brane construction relates a quiver with all ranks equal on one side, and another quiver with different ranks on the other side. In the following we will verify that this is a toric duality by computing and comparing the branch $\mathcal{M}_{4}$ of the abelian moduli space (i.e. the toric diagram) of the two dual descriptions. The match of $\mathcal{M}_{4}$ is just a check of the proposed duality, and we do not intend to use this tool to claim other dualities than the ones obtained generalizing the brane picture. Indeed at this level other dualities are possible. For example the initial quiver with equal ranks has the same $\mathcal{M}_{4}$ than the same quiver but with different ranks. However these models are presumably not equivalent despite sharing the same $\mathcal{M}_{4}$. Since $\mathcal{M}_{4}$ corresponds to the classical abelian moduli space, it is plausible that we cannot capture some feature of the full non abelian dynamics of the theory. In particular a complete analysis of the field theory moduli space or of the dual geometric background would be interesting. We leave this analysis for future studies. 


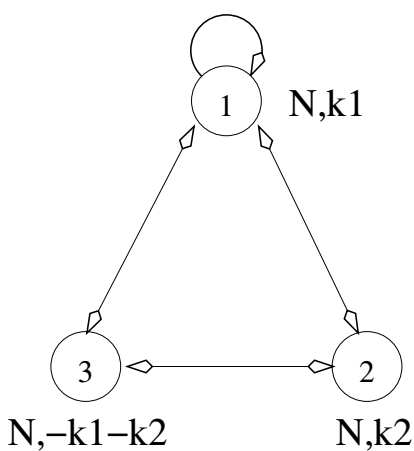

(a)

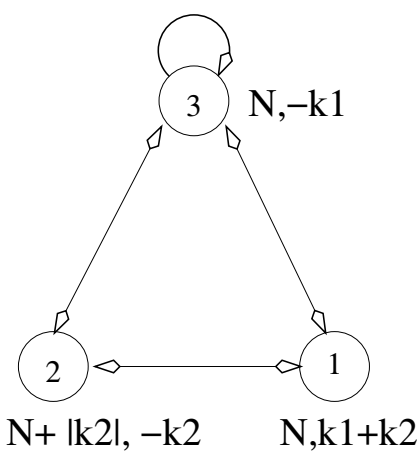

(b)

Figure 4. Quiver, ranks and CS levels for the $\widetilde{L^{121}}\left\{k_{1}, k_{2}, k_{3}\right\}$ in the two phases related by Seiberg like duality on node 2 .

As observed, the Seiberg like duality (3.4) modifies the rank of the dualized gauge group, introducing fractional branes. This is a novelty of this $3 d$ duality with respect to the $4 d$ case (see also $[34,35,50]$ ). A first consistency check of the transformation (3.4) involving $\left|k_{i}\right|$ fractional brane is that performing another Seiberg-like duality on the same node leads back to the original electric theory.

The $k$ fractional D3 branes are stuck between the five branes, so there is no moduli space associated with their motion. This is as discussed in [34], and the same field theory argument can be repeated here. The classical moduli space of the magnetic description is then the $N$ symmetric product of the abelian moduli space.

The fractional branes can break supersymmetry as a consequence of the s-rule [45]. Indeed it was suggested in $[47,51,52]$ that for $\mathrm{U}(l)_{k}$ YM-CS theories supersymmetry is broken if $l>|k|$. We notice from (3.4) that in the moduli space of the magnetic description, there is a pure $\mathrm{U}(k)_{-k}$ YM-CS theory. Thus the bound is satisfied and supersymmetry is unbroken. However, if we perform multiple dualities we can realize configurations with several fractional branes on different nodes. At every duality we have to control via srule that supersymmetry is not broken. A more thorough study of these issues related to fractional branes is beyond the scope of the present paper.

Finally, the duality proposed maps a theory with a weak coupling limit to a strongly coupled theory. Indeed if we define the $i$-th 't Hooft coupling as $\lambda_{i}=N / k_{i}$, the original theory is weakly coupled for $k_{i} \gg N$. In this limit the dual theory is strongly coupled since the i-th dual 't Hooft coupling is $\tilde{\lambda}_{i}=1+O\left(N / k_{i}\right)$.

\section{$3.2 \widetilde{L^{121}}\left\{k_{1}, k_{2}, k_{3}\right\}$}

The $\widetilde{L^{121}}\left\{k_{1}, k_{2}, k_{3}\right\}$ is the first example that we study. The quiver is given in figure 4 . The toric diagram that encodes the information about the classical mesonic moduli space is computed with the techniques explained in section 2 . We extract the incidence matrix $d_{G, i}$, where $G=1, \ldots, N_{g}$ runs over the labels of the gauge groups, and $i$ runs over the 
fields ( $i=1, \ldots, 7$ for the ${\widetilde{L^{121}}}_{\left\{k_{1}, k_{2}, k_{3}\right\}}$, six bifundamental and one adjoint)

$$
d=\left(\begin{array}{ccccccc}
1 & -1 & 0 & 0 & -1 & 1 & 0 \\
-1 & 1 & 1 & -1 & 0 & 0 & 0 \\
0 & 0 & -1 & 1 & 1 & -1 & 0
\end{array}\right)
$$

The matrix of the perfect matchings $P_{\alpha, i}$ is computed from the determinant of the Kastelein matrix

$$
K a s=\left(\begin{array}{cc}
Q_{23}+Q_{32} & Q_{12}+Q_{21} \\
Q_{13}+Q_{31} & X_{11}
\end{array}\right)
$$

If we order the fields in the determinant of (3.6) we can build the matrix $P_{i, \alpha}$ where $\alpha=1, \ldots, c$ is the number of perfect matchings, that corresponds to the number of monomials of the det Kas. In this matrix we have 1 if the $i$-th field appears in the $\alpha$-th element of the determinant, 0 otherwise

$$
P=\left(\begin{array}{llllll}
1 & 0 & 0 & 0 & 1 & 0 \\
0 & 1 & 0 & 0 & 0 & 1 \\
0 & 0 & 0 & 1 & 0 & 0 \\
0 & 0 & 1 & 0 & 0 & 0 \\
0 & 1 & 0 & 0 & 1 & 0 \\
1 & 0 & 0 & 0 & 0 & 1 \\
0 & 0 & 1 & 1 & 0 & 0
\end{array}\right)
$$

The matrix $Q$ that represents the charge matrix for the GLSM fields is obtained from the relation $d_{G, i}=Q_{G, \alpha} \cdot P_{i, \alpha}^{T}$

$$
Q=\left(\begin{array}{cccccc}
1 & -1 & 0 & 0 & 0 & 0 \\
0 & 1 & -1 & 1 & -1 & 0 \\
-1 & 0 & 1 & -1 & 1 & 0
\end{array}\right)
$$

The contribution of the $D$-terms to the moduli space is given by quotienting by the $G-2$ FI parameters induced by the CS couplings. These FI parameters are in the integer kernel of the matrix of the CS level

$$
K=\operatorname{Ker}\left(\begin{array}{ccc}
1 & 1 & 1 \\
k_{1} & k_{2} & -k_{1}-k_{2}
\end{array}\right)
$$

The $F$-term equations are encoded in the matrix $Q_{F}=\operatorname{Ker}(P)$. The Toric diagram is the kernel of the matrix obtained by combining $Q_{D}=K \cdot Q$ and $Q_{F}$. Acting with an $\operatorname{SL}(4, Z)$ transformation the toric diagram reads

$$
G_{t}=\operatorname{Ker}^{*}\left[K \cdot Q, \operatorname{Ker}\left[P^{T}\right]\right]=\left(\begin{array}{ccccccc}
1 & 1 & 1 & 1 & 1 & 1 \\
k_{2} & k_{1}+k_{2} & 0 & 0 & k_{1}+2 k_{2} & 0 \\
1 & 1 & 0 & 1 & 2 & 0 \\
0 & 0 & 1 & 1 & 0 & 0
\end{array}\right)
$$




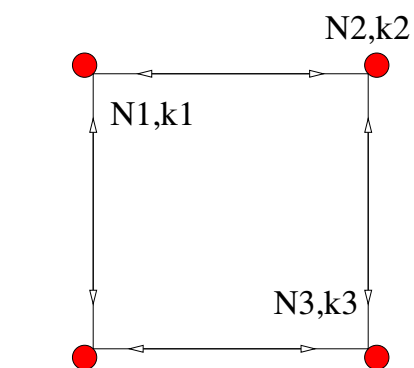

$\mathrm{N} 4,-\mathrm{k} 1-\mathrm{k} 2-\mathrm{k} 3$

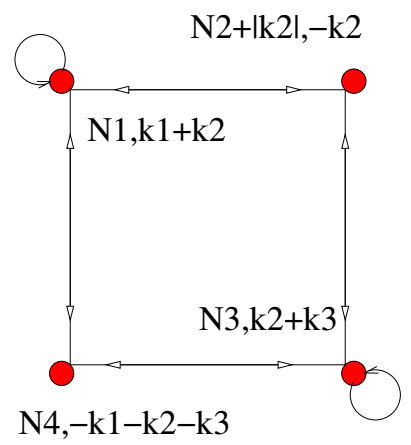

$\mathrm{N} 4,-\mathrm{k} 1-\mathrm{k} 2-\mathrm{k} 3$

Figure 5. The quivers for the two phases of $L^{222}$.

This system of vectors is co-spatial. This is a CY condition that guarantees that the toric diagram lives on a three dimensional hypersurface in $Z_{4}$.

The last three rows of (3.10) defines the toric diagram for the three dimensional ChernSimons $\left.\widetilde{L^{121}} k_{\left\{k_{1}\right.}, k_{2}, k_{3}\right\}$ toric quiver gauge theory. Note that the toric diagram of the $(3+1) \mathrm{d}$ parent is recovered by setting to zero the row with the CS levels.

We perform the Seiberg-like duality (3.4) on node 2. The resulting theory is shown in figure 4(b). The $L^{121}$ four dimensional parent theory has only one toric phase. The dual theory is in the same phase, thanks to the mapping among the nodes $(1 \rightarrow 3,2 \rightarrow 1,3 \rightarrow 2)$, see figure 4. In the $\widehat{L}^{121}\left\{k_{1}, k_{2}, k_{3}\right\}$ we should also properly map the CS levels in the two dual descriptions. The transformation rules (3.4) change the CS level as in figure 4. Then we apply the same mapping we used for the gauge groups. After these steps the resulting $K$ matrix is

$$
K_{\text {dual }}=\operatorname{Ker}\left(\begin{array}{ccc}
1 & 1 & 1 \\
\tilde{k}_{3} & \tilde{k}_{1} & \tilde{k}_{2}
\end{array}\right)=\operatorname{Ker}\left(\begin{array}{ccc}
1 & 1 & 1 \\
k_{3}+k_{2} & k_{1}+k_{2} & -k_{2}
\end{array}\right)=\operatorname{Ker}\left(\begin{array}{ccc}
1 & 1 & 1 \\
-k_{1} & k_{1}+k_{2} & -k_{2}
\end{array}\right)
$$

where with $\tilde{k}_{i}$ we denote the CS level of the $i$-th node in the dual phase. Concerning the field content and the superpotential, the dual theory is in the same phase than the starting theory. Thus we use the same matrices $P, d, Q$ for the computation of the moduli space. The toric diagram is then computed with the usual algorithm. Up to an $\operatorname{SL}(4, Z)$ transformation it coincides with the same as the one computed in the original theory (3.10).

In this example we have shown that the Seiberg like duality (3.4) is a toric duality. Observe that the non trivial transformation on the CS levels of (3.4) are necessary for the equivalence of the moduli spaces of the two phases.

\section{$3.3 \widetilde{L^{222}}\left\{k_{i}\right\}$}

The second example is the $\widetilde{L^{222}}\left\{k_{i}\right\}$ theory. The main difference is that this theory has two phases with a different matter content and superpotential (see figure 5), obtained by dualizing node 2 . These phases are dual for the $(3+1) d$ parents theory. Here we show that the same holds in three dimensions with the Seiberg like duality (3.4). The $P, d, Q$ and $K$ 
matrices for the first phase are

$$
\begin{aligned}
& d=\left(\begin{array}{cccccccc}
1 & -1 & 0 & 0 & 0 & 0 & -1 & 1 \\
-1 & 1 & 1 & -1 & 0 & 0 & 0 & 0 \\
0 & 0 & -1 & 1 & 1 & -1 & 0 & 0 \\
0 & 0 & 0 & 0 & -1 & 1 & 1 & -1
\end{array}\right) \quad P=\left(\begin{array}{llllllll}
0 & 0 & 0 & 0 & 1 & 0 & 1 & 0 \\
0 & 0 & 0 & 0 & 0 & 1 & 0 & 1 \\
1 & 0 & 1 & 0 & 0 & 0 & 0 & 0 \\
0 & 1 & 0 & 1 & 0 & 0 & 0 & 0 \\
0 & 0 & 0 & 0 & 1 & 1 & 0 & 0 \\
0 & 0 & 0 & 0 & 0 & 0 & 1 & 1 \\
1 & 1 & 0 & 0 & 0 & 0 & 0 & 0 \\
0 & 0 & 1 & 1 & 0 & 0 & 0 & 0
\end{array}\right) \\
& Q=\left(\begin{array}{cccccccc}
-1 & 0 & 1 & 0 & 1 & -1 & 0 & 0 \\
1 & -1 & 0 & 0 & -1 & 1 & 0 & 0 \\
-1 & 1 & 0 & 0 & 0 & 1 & 0 & -1 \\
1 & 0 & -1 & 0 & 0 & -1 & 0 & 1
\end{array}\right) \quad K=\operatorname{Ker}\left(\begin{array}{ccccc}
1 & 1 & 1 & \\
k_{1} & k_{2} & k_{3} & -k_{1}-k_{2}-k_{3}
\end{array}\right) \\
& 1
\end{aligned}
$$

The resulting toric diagram is

$$
G_{t}=\left(\begin{array}{cccccccc}
1 & 1 & 1 & 1 & 1 & 1 & 1 & 1 \\
2 & 1 & 1 & 0 & 2 & 1 & 1 & 0 \\
1 & 1 & 1 & 1 & 0 & 0 & 0 & 0 \\
-k 1-k_{2} & -k_{1} & k_{2} & 0 & k_{2}+k_{3} & k_{2}+k_{3} & 0 & 0
\end{array}\right)
$$

By duality on node 2 we obtain the inequivalent phase of $\widetilde{L^{222}}\left\{k_{i}\right\}$, see figure 5 . The toric diagram is computed with new $d, P, Q$ and $K$ matrices

$$
\begin{aligned}
& d=\left(\begin{array}{cccccccccc}
0 & 1 & -1 & 0 & 0 & 0 & 0 & 0 & -1 & 1 \\
0 & -1 & 1 & 1 & -1 & 0 & 0 & 0 & 0 & 0 \\
0 & 0 & 0 & -1 & 1 & 0 & 1 & -1 & 0 & 0 \\
0 & 0 & 0 & 0 & 0 & 0 & -1 & 1 & 1 & -1
\end{array}\right) \quad P=\left(\begin{array}{cccccccc}
0 & 0 & 0 & 0 & 1 & 1 & 1 & 1 \\
1 & 0 & 1 & 0 & 0 & 0 & 0 & 0 \\
0 & 1 & 0 & 1 & 0 & 0 & 0 & 0 \\
0 & 0 & 0 & 0 & 1 & 0 & 1 & 0 \\
0 & 0 & 0 & 0 & 0 & 1 & 0 & 1 \\
1 & 1 & 1 & 1 & 0 & 0 & 0 & 0 \\
0 & 0 & 0 & 0 & 1 & 1 & 0 & 0 \\
0 & 0 & 0 & 0 & 0 & 0 & 1 & 1 \\
1 & 1 & 0 & 0 & 0 & 0 & 0 & 0 \\
0 & 0 & 1 & 1 & 0 & 0 & 0 & 0
\end{array}\right) \\
& Q=\left(\begin{array}{cccccccc}
0 & -1 & 1 & 0 & 0 & 0 & 0 & 0 \\
0 & 0 & -1 & 1 & 0 & 0 & 1 & -1 \\
0 & 0 & 0 & 0 & 0 & 1 & -1 & 0 \\
0 & 1 & 0 & -1 & 0 & -1 & 0 & 1
\end{array}\right) \quad K=K \operatorname{Ker}\left(\begin{array}{cccccc}
1 \\
k_{1}+k_{2}
\end{array}\right) \\
& 1
\end{aligned}
$$

It results

$$
G_{t}=\left(\begin{array}{cccccccc}
1 & 1 & 1 & 1 & 1 & 1 & 1 & 1 \\
2 & 1 & 1 & 0 & 2 & 1 & 1 & 0 \\
-k 1-k_{2} & -k_{1} & k_{2} & 0 & k_{2}+k_{3} & k_{2}+k_{3} & 0 & 0 \\
1 & 1 & 1 & 1 & 0 & 0 & 0 & 0
\end{array}\right)
$$

which is equivalent to (3.14). 


\subsection{The general $\widetilde{L^{a b a}}\left\{k_{i}\right\}$}

In the previous section we have seen two simple examples. In this section we consider the generic case of non-chiral $\mathcal{N}=2$ toric three dimensional CS quiver gauge theories $\widetilde{L^{a b a}}\left\{k_{i}\right\}$ singularities. Four dimensional theories based on these singularities share the same toric diagram among the different Seiberg dual phases. Here we show that two theories that are related by Seiberg like duality in three dimension (3.4) share the same toric diagram. Our argument is based on the algorithm [30] that extracts toric data of the CY four-fold by using brane tiling.

\section{Toric diagrams from bipartite graphs}

Let us remind the reader that to every quiver describing a four dimensional conformal field theory on D3 branes at Calabi Yau three-fold singularities it is possible to associate a bipartite diagram drawn on a torus. It is called tiling or dimer $[53,54]$, and it encodes all the informations in the quiver and in the superpotential. To every face in the dimer we can associate a gauge group factor, to every edge a bifundamental field and to every node a term in the superpotential. A similar tiling can be associated with three dimensional ChernSimons matter theories living on M2 branes probing a Calabi Yau four-fold singularity simply adding a flux of Chern-Simons charge. The CS levels are described as a conserved flow on the quiver, or equivalently on the dimer. To every edge we associate a flux of Chern-Simons charge and the CS level of the gauge group is the sum of these contributions taken with sign depending on the orientation of the arrow.

In this section we review the proposal of [30] for the computation of the moduli space of three dimensional CS toric quiver gauge theories. This method furnishes the toric data from the bipartite graphs associated with the quiver model and with the CS levels.

One has to choose a set of paths $\left(p_{1}, \ldots, p_{4}\right)$ on the dimer. The paths $p_{1}$ and $p_{2}$ correspond to the $\alpha$ and $\beta$ cycles of the torus described by the dimer. The path $p_{4}$ is a paths encircling one of the vertexes. One can also associate mesonic operators to these paths. These operators correspond to the product of the corresponding bifundamentals along the paths. For example the operator associated on the $p_{4}$ path is a term of the superpotential. The moduli space of the three dimensional theory requires also the definition of the path $p_{3}$. This is a product of paths corresponding to a closed flow of CS charges along the quiver. We choose $p_{3}$ in the tiling of the $L^{a b a}$ singularity by taking a minimal closed path connecting the bifundamentals from the first node to the $a+b$-th node of the quiver. Then one associates the CS charge

$$
\sum_{i=1}^{n} k_{i}
$$

to each bifundamental in this closed loop, with $n=1, \ldots, a+b$. The last charge is zero since it corresponds to sum of all the CS levels in the theory. This conserved CS charges flow is represented on the dimer by a set of oriented arrows connecting the faces of the dimer, the gauge groups. 


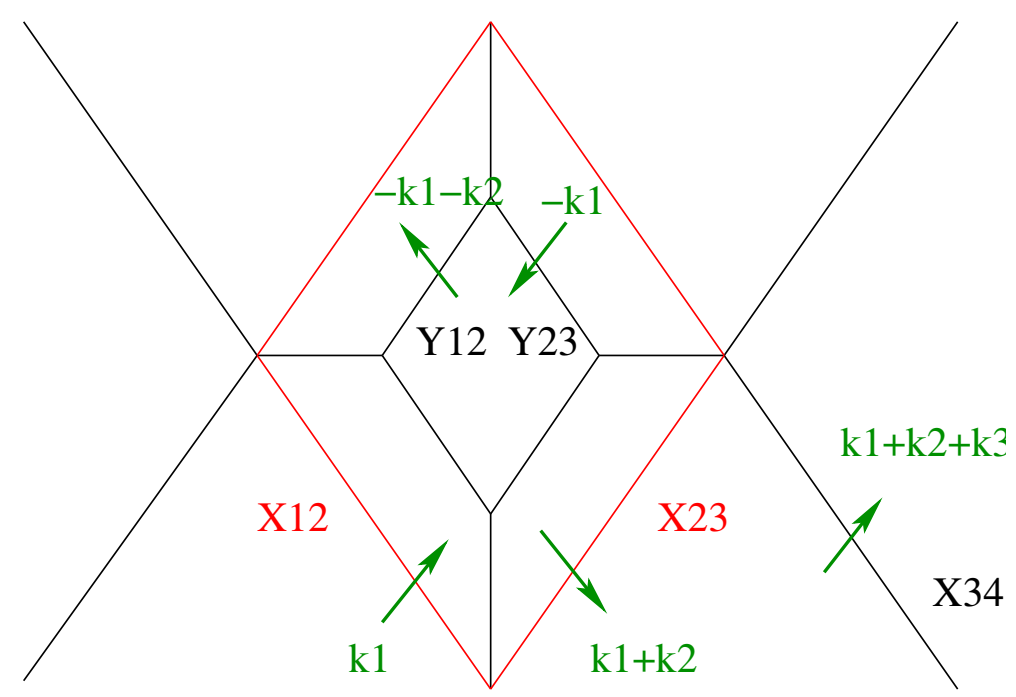

Figure 6. Action of Seiberg duality on the dimer and modification of the CS flow.

In [30] it has been shown that the toric polytope of $C Y_{4}$ is given by the convex hull of all lattice point $v^{\alpha}=\left(v_{1}^{\alpha}, \ldots, v_{4}^{\alpha}\right)$, with

$$
v_{i}^{\alpha}=\left\langle p_{i}, D_{\alpha}\right\rangle
$$

where $D_{\alpha}$ are the perfect matchings (see [53] for a definition of perfect matchings in dimers). The operation $\langle.,$.$\rangle in (3.19) is the signed intersection number of the perfect matching D_{\alpha}$ with the path $p_{i}$. Note that $v_{4}$ is always 1 , since there is always only one perfect matching connected with the node encircled by $p_{4}$.

\section{Seiberg-like duality on $\widetilde{L^{a b a}}\left\{k_{i}\right\}$ and toric duality}

In subsection (3.1) we argued that the action of Seiberg duality on the field content and on the superpotential is the same as in four dimensions. The only difference is the change of the CS levels associated with the groups involved in the duality (3.4).

In an $L^{a b a}$ theory, if duality is performed on node $N_{2}$, the levels become

$$
\begin{aligned}
& k_{1} \rightarrow k_{1}+k_{2} \\
& k_{2} \rightarrow-k_{2} \\
& k_{3} \rightarrow k_{3}+k_{2}
\end{aligned}
$$

The action of Seiberg duality (3.4) not only modifies the dimer as in the $4 \mathrm{~d}$, but also the CS levels of the gauge groups. This changes the path $p_{3}$ in the dual description. Many $\mathrm{SL}(4, Z)$ equivalent choices are possible. Among them we select the $p_{3}$ path as in figure 6 . We associate a charge $l_{1}$ to $Y_{12}$ and $l_{2}$ to $Y_{23}$, but with the opposite arrows that before. The values of the charges $l_{1}$ and $l_{2}$ are derived from (3.20) and are

$$
l_{1}=-k_{1}-k_{2} \quad l_{2}=-k_{1}
$$



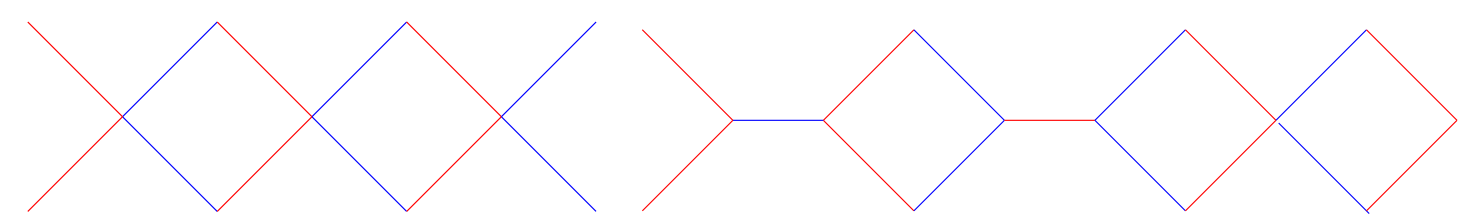

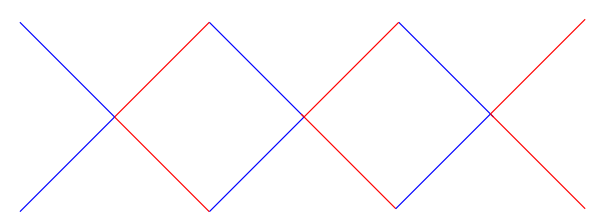

(a)

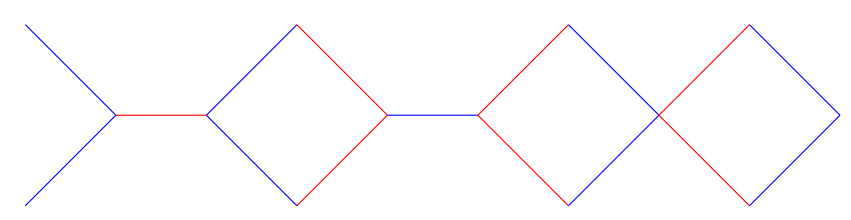

(b)

Figure 7. Different sectors of fields that generate all perfect matching in the (a) electric and (b) magnetic theory

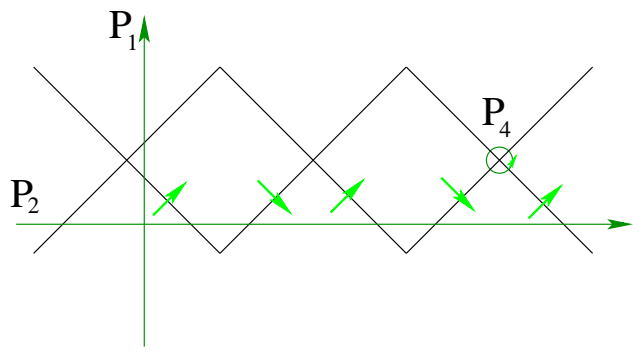

(a)

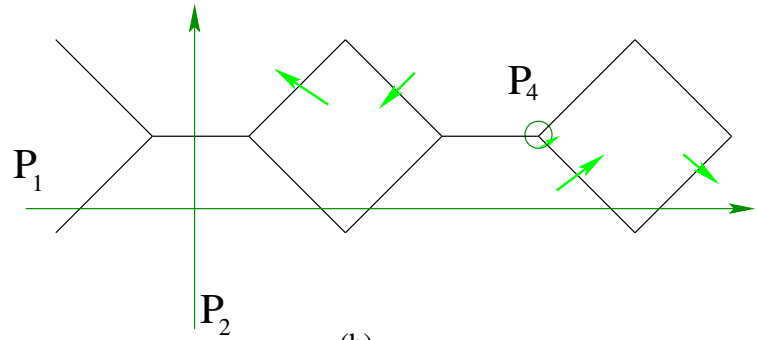

(b)

Figure 8. Paths $p_{i}$ in the two dual versions of the theory

The field $X_{34}$ is not involved in this duality and it contributes to the CS flow with the same charge $k_{1}+k_{2}+k_{3}$ in both phases.

We claim that the two theories share the same toric diagram. For the proof of this relation it is useful to distinguish two sector of fields from which all the perfect matching are built. In figure 7 we separated these two sectors for the electric and magnetic phase of an $L^{a a a}$ theory (the same distinction is possible in a generic $L^{a b a}$ theory). Every perfect matching is built by choosing in these sets only one field associated with each vertex. For example in figure 7(a) every perfect matching is a set of blue lines chosen such that every vertex is involved only once.

The paths $p_{1}$ and $p_{2}$ are shown in figure 8 for the electric and the magnetic phase. The intersection numbers $\left\langle p_{1}, D^{\alpha}\right\rangle$ and $\left\langle p_{2}, D^{\alpha}\right\rangle$ give the same bi-dimensional toric diagram of the associated four dimensional $L^{a b a}$ theories, up to an overall $\mathrm{SL}(3, Z)$ translation. This is shown by mapping the perfect matching in the two description. This mapping is done with a prescription on the choice of the fields in the perfect matching of the dual description. If duality is performed on node $N_{i}$, the field $X_{i-1, i}, X_{i, i-1}, X_{i, i+1}$ and $X_{i+1, i}$ are respectively mapped in the fields $Y_{i, i+1}, Y_{i+1, i}, Y_{i-1, i}$ and $Y_{i . i-1}$ of the dual theory. This prescription 

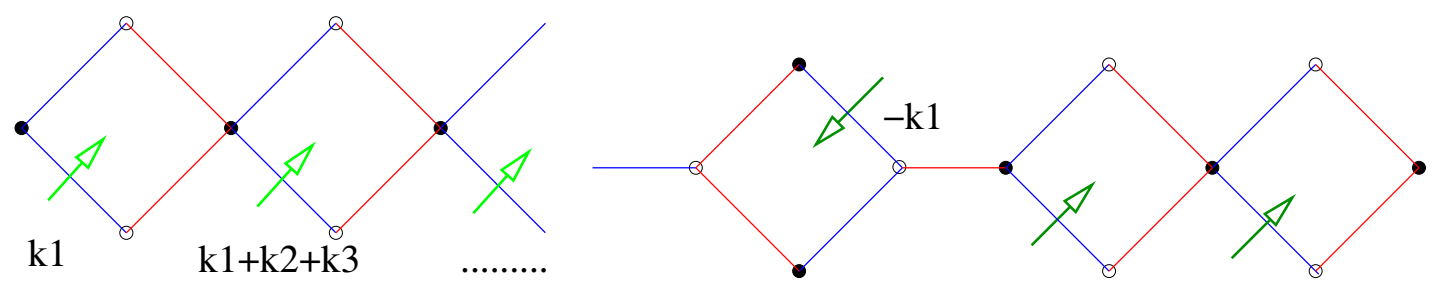

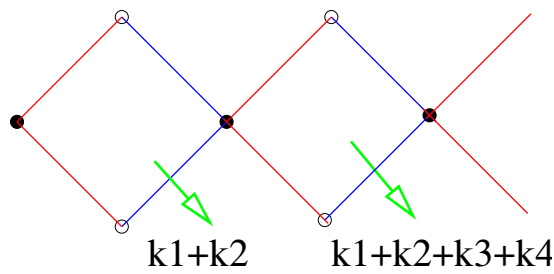

(a)

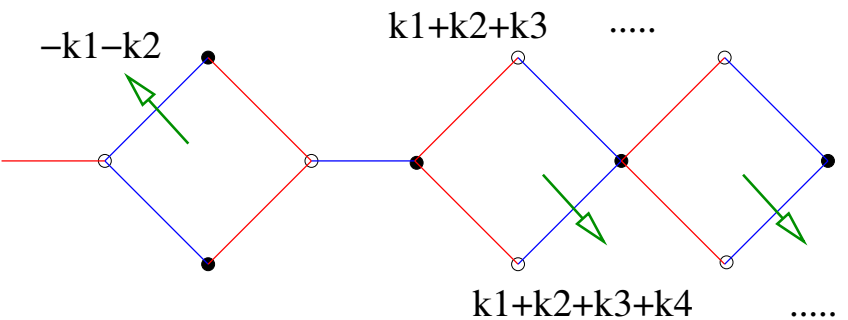

(b)

Figure 9. Decomposition of the path $p_{3}$ on the different perfect matchings

gives a $1-1$ map of each point of the $2 \mathrm{~d}$ toric diagrams of the two dual theories.

The whole diagram for the three dimensional $\widetilde{L^{a b a}}$ theory is obtained by considering the intersection numbers $\left\langle p_{3}, D^{\alpha}\right\rangle$, which give the third component of the vectors $v^{\alpha}$. The path $p_{3}$ corresponds to the flux of CS charges from the group $N_{1}$ to the group $N_{g}$, where the last arrow is omitted since it carries zero charge. In the dual theory the path $p_{3}$ changes as explained above, and as we show in figure 8 for duality on a node labeled by 2 . Note that only the arrows connected with the dualized gauge group change.

With this choice of $p_{3}$ and using the basis of perfect matching we prescribed, the intersection numbers $\left\langle p_{3}, D^{\alpha}\right\rangle$ coincide in the two phases for every point of the $2 \mathrm{~d}$ toric diagram. This is shown by associating the relevant part of the path $p_{3}$ to each sector of perfect matching as in figure 9 . The arrow that carries charge $k_{1}$ in the electric theory corresponds to the arrow with charge $-k_{1}$ in the magnetic theory. Its contribution to the moduli space remains the same, since also the orientation of this arrow is the opposite. The same happens for the arrow carrying charge $k_{1}+k_{2}$.

Thus the $3 \mathrm{~d}$ toric diagrams of the two dual theories are the same. We conclude that the action of three dimensional Seiberg like duality (3.4) in the $\widetilde{L^{a b a}}\left\{k_{i}\right\}$ theories implies toric duality.

\section{Chiral theories}

In this section we study Seiberg like duality for $\mathcal{N}=2$ three dimensional Chern-Simons theories with four dimensional parent chiral theories, which as such suffer from anomalies. The anomaly free condition imposes constraints on the rank distribution. In $3 \mathrm{~d}$ there are no local gauge anomalies. Nevertheless we work with all $\mathrm{U}(N)$ gauge groups such that the moduli space is the $N$ symmetric product of the abelian one. Moreover for three dimensional Chern-Simons chiral theories we do not have a brane construction as simple as for the non-chiral case, and were not able to deduce the duality from the brane picture. 


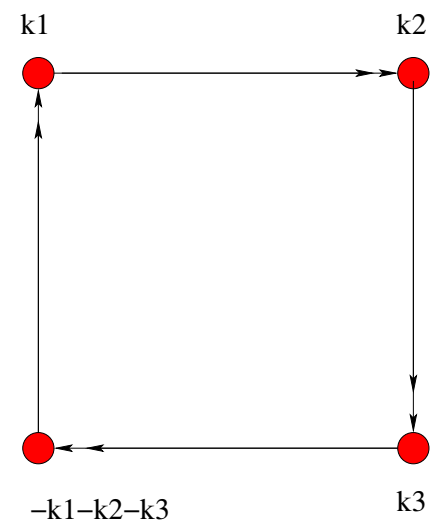

(a)

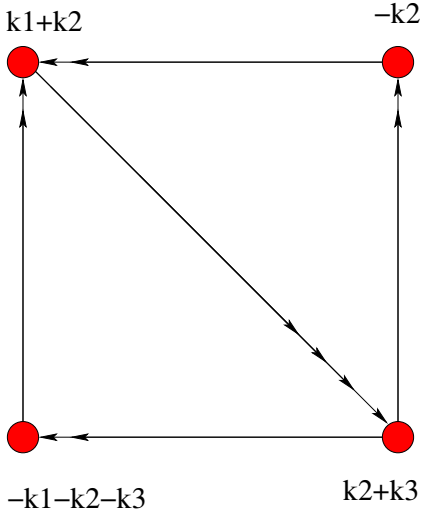

(b)

Figure 10. (a) quiver for $\widetilde{\mathbb{F}_{0}^{I}}$ and (b) quiver for $\widetilde{\mathbb{F}_{0}^{I I}}$ for one of the possible choice of CS levels.

However, using what we learned from the non-chiral case, we infer that at least a subset of the possible three dimensional Seiberg-like toric dualities acts on the field content and on the superpotential as it does in $4 \mathrm{~d}$ and moreover recombines the Chern-Simons levels in a similar way as in (3.4). As a matter of fact, a straightforward extension of the rule (3.4) does not seem to work in the chiral case. This could be related to the fact that the Master Spaces of the four dimensional dual Seiberg parents are not isomorphic [41]. For this reason we restrict ourself to the case where the CS level of the group which undergoes duality is set to zero. We assume that the other CS levels are unchanged, and no fractional branes are created. This could be suggested by the parity anomaly matching argument (see appendix A). We also set to zero the CS level of those gauge groups that after duality have the same interactions with the rest of the quiver as the dualized gauge group.

By direct inspection we find that under these assumptions on the CS levels also for chiral 3D CS theories Seiberg like duality leads to toric duality. For $\widetilde{\mathbb{F}_{0}}$ we can take milder assumptions. Indeed we find that a generalization of the rule (3.4) to the chiral case still gives toric duality for this theory.

\section{$4.1 \widetilde{\mathbb{F}_{0}}\left\{k_{i}\right\}$}

Here we study the $(2+1)$ d CS chiral theory whose $(3+1) d$ parent is $\mathbb{F}_{0}$. In $(3+1) d$ there are two dual toric phases of $\mathbb{F}_{0}$, denoted as $\mathbb{F}_{0}^{I}$ and $\mathbb{F}_{0}^{I I}$. In $(2+1)$ d the two phases for arbitrary choices of the CS levels do not have the same moduli space. Nevertheless it is possible to find assignments for the Chern-Simons levels such that the two phases have the same toric diagram.

The quiver representing the $\widetilde{\mathbb{F}_{0}^{I}}$ phase is in figure $10(\mathrm{a})$. The superpotential is

$$
W=\varepsilon_{i j} \varepsilon_{k l} X_{12}^{i} X_{23}^{k} X_{34}^{j} X_{41}^{l}
$$


The incidence matrix and the matrix of perfect matchings are

$$
d=\left(\begin{array}{cccccccc}
1 & -1 & 0 & 0 & 0 & 0 & -1 & 1 \\
-1 & 1 & 1 & -1 & 0 & 0 & 0 & 0 \\
0 & 0 & -1 & 1 & 1 & -1 & 0 & 0 \\
0 & 0 & 0 & 0 & -1 & 1 & 1 & -1
\end{array}\right) \quad P=\left(\begin{array}{cccccccc}
1 & 1 & 0 & 0 & 0 & 0 & 0 & 0 \\
1 & 0 & 1 & 0 & 0 & 0 & 0 & 0 \\
0 & 0 & 0 & 0 & 1 & 1 & 0 & 0 \\
0 & 0 & 0 & 0 & 1 & 0 & 1 & 0 \\
0 & 1 & 0 & 1 & 0 & 0 & 0 & 0 \\
0 & 0 & 1 & 1 & 0 & 0 & 0 & 0 \\
0 & 0 & 0 & 0 & 0 & 1 & 0 & 1 \\
0 & 0 & 0 & 0 & 0 & 0 & 1 & 1
\end{array}\right)
$$

The charges of the GLSM fields determine the matrix Q, the can be chosen as

$$
Q=\left(\begin{array}{cccccccc}
1 & 0 & 0 & 0 & 0 & 0 & 0 & -1 \\
-1 & 0 & 0 & 0 & 1 & 0 & 0 & 0 \\
0 & 0 & 0 & 1 & -1 & 0 & 0 & 0 \\
0 & 0 & 0 & -1 & 0 & 0 & 0 & 1
\end{array}\right)
$$

The second phase $\widetilde{\mathbb{F}_{0}^{I I}}$ is obtained by dualizing node 2 . The dual superpotential is

$$
W=\varepsilon_{i j} \varepsilon_{k l} X_{13}^{i k} X_{32}^{l} X_{21}^{j}-\varepsilon_{i j} \varepsilon_{k l} X_{13}^{i k} X_{34}^{l} X_{41}^{j}
$$

The matrices $d, P$ and $Q$ are determined from the quiver and the superpotential

$$
\begin{aligned}
d & =\left(\begin{array}{cccccccccccc}
-1 & -1 & 0 & 0 & 0 & 0 & -1 & -1 & 1 & 1 & 1 & 1 \\
1 & 1 & -1 & -1 & 0 & 0 & 0 & 0 & 0 & 0 & 0 & 0 \\
0 & 0 & 1 & 1 & 1 & 1 & 0 & 0 & -1 & -1 & -1 & -1 \\
0 & 0 & 0 & 0 & -1 & -1 & 1 & 1 & 0 & 0 & 0 & 0
\end{array}\right) \\
P & =\left(\begin{array}{ccccccccc}
0 & 0 & 0 & 0 & 0 & 1 & 0 & 1 & 1 \\
0 & 0 & 1 & 0 & 0 & 1 & 0 & 0 & 1 \\
0 & 0 & 0 & 1 & 1 & 0 & 1 & 0 & 0 \\
0 & 1 & 0 & 0 & 1 & 0 & 1 & 0 & 0 \\
0 & 0 & 1 & 0 & 0 & 0 & 1 & 0 & 1 \\
0 & 0 & 0 & 0 & 0 & 0 & 1 & 1 & 1 \\
0 & 1 & 0 & 0 & 1 & 1 & 0 & 0 & 0 \\
0 & 0 & 0 & 1 & 1 & 1 & 0 & 0 & 0 \\
1 & 1 & 1 & 0 & 0 & 0 & 0 & 0 & 0 \\
1 & 1 & 0 & 0 & 0 & 0 & 0 & 1 & 0 \\
1 & 0 & 0 & 1 & 0 & 0 & 0 & 1 & 0 \\
1 & 0 & 1 & 1 & 0 & 0 & 0 & 0 & 0
\end{array}\right) \\
Q & =\left(\begin{array}{ccccccccc}
1 & 0 & 0 & 0 & 0 & -1 & 0 & 0 & 0 \\
0 & 0 & 0 & 0 & -1 & 1 & 0 & 0 & 0 \\
-1 & 0 & 0 & 0 & 0 & 0 & 1 & 0 & 0 \\
0 & 0 & 0 & 0 & 1 & 0 & -1 & 0 & 0
\end{array}\right)
\end{aligned}
$$




\section{Two families}

The $\widetilde{\left.\mathbb{F}_{0\{k}\right\}}$ theories turn out to be a special case. Indeed one can single out two different possibilities: in the first one we put to zero just the CS level associated with the group 2; while in the second case we can fix to zero just the Chern-Simons level of the group 4 and transform the CS levels as in the non-chiral case. In the first case we choose the CS levels as $\left(k_{1}, k_{2}, k_{3}, k_{4}\right)=(k, 0, p,-k-p)$. The CS level matrix for both phases is:

$$
C=\left(\begin{array}{cccc}
1 & 1 & 1 & 1 \\
k & 0 & p & -k-p
\end{array}\right)
$$

and the toric diagram is given by:

$$
G_{t}^{(I)}=\left(\begin{array}{cccccccc}
1 & 1 & 1 & 1 & 1 & 1 & 1 & 1 \\
\mathrm{p} & 0 & \mathrm{p} & 0 & \mathrm{p} & \mathrm{p} & \mathrm{k}+\mathrm{p} & \mathrm{k}+\mathrm{p} \\
0 & 1 & -1 & 0 & 0 & 0 & 0 & 0 \\
0 & 0 & 0 & 0 & 0 & 1 & -1 & 0
\end{array}\right)
$$

The CS levels for the dual phase, obtained by duality on $N_{2}$, are unchanged and the toric diagram

$$
G_{t}^{(I I)}=\left(\begin{array}{ccccccccc}
1 & 1 & 1 & 1 & 1 & 1 & 1 & 1 & 1 \\
\mathrm{p} & \mathrm{k}+\mathrm{p} & \mathrm{p} & \mathrm{p} & \mathrm{k}+\mathrm{p} & \mathrm{k}+\mathrm{p} & 0 & 0 & 0 \\
0 & 0 & -1 & 0 & 0 & 0 & 0 & 1 & 0 \\
0 & -1 & 0 & 1 & 0 & 0 & 0 & 0 & 0
\end{array}\right)
$$

is equivalent to the one above.

In the second case we choose the CS levels as $\left(k_{1}, k_{2}, k_{3}, k_{4}\right)=(k, p,-k-p, 0)$. The phase $\widetilde{\mathbb{F}_{0}^{I I}}$ is computed by dualizing the node 2 . We observe that by applying the rules (3.4) the CS levels of $\widetilde{\mathbb{F}_{0}^{I I}}$ are $(k+p,-p,-k, 0)$. The $C$ matrices for the two phases are:

$$
C_{I}=\left(\begin{array}{cccc}
1 & 1 & 1 & 1 \\
k & p & -k-p & 0
\end{array}\right) \quad C_{I I}=\left(\begin{array}{cccc}
1 & 1 & 1 & 1 \\
k+p & -p & -k & 0
\end{array}\right)
$$

The toric diagram for the first phase is:

$$
G_{t}^{(I)}=\left(\begin{array}{cccccccc}
1 & 1 & 1 & 1 & 1 & 1 & 1 & 1 \\
\mathrm{k} & \mathrm{k} & 0 & 0 & \mathrm{k}+\mathrm{p} & 0 & \mathrm{k}+\mathrm{p} & 0 \\
0 & 0 & 0 & 0 & 0 & 1 & -1 & 0 \\
0 & -1 & 1 & 0 & 0 & 0 & 0 & 0
\end{array}\right)
$$

while the toric diagram for the second phase is:

$$
G_{t}^{(I I)}=\left(\begin{array}{ccccccccc}
1 & 1 & 1 & 1 & 1 & 1 & 1 & 1 & 1 \\
0 & 0 & 0 & \mathrm{k} & \mathrm{k} & \mathrm{k}+\mathrm{p} & \mathrm{k} & \mathrm{k}+\mathrm{p} & \mathrm{k}+\mathrm{p} \\
0 & 0 & 1 & 0 & 0 & 0 & 0 & -1 & 0 \\
0 & 1 & 0 & -1 & 0 & 0 & 0 & 0 & 0
\end{array}\right)
$$

And they are equivalent.

The $\mathbb{F}_{0}$ theory seems to be the only case where the assumptions we gave at the beginning of this section can be relaxed. In the following examples we will just apply those basic rules. 

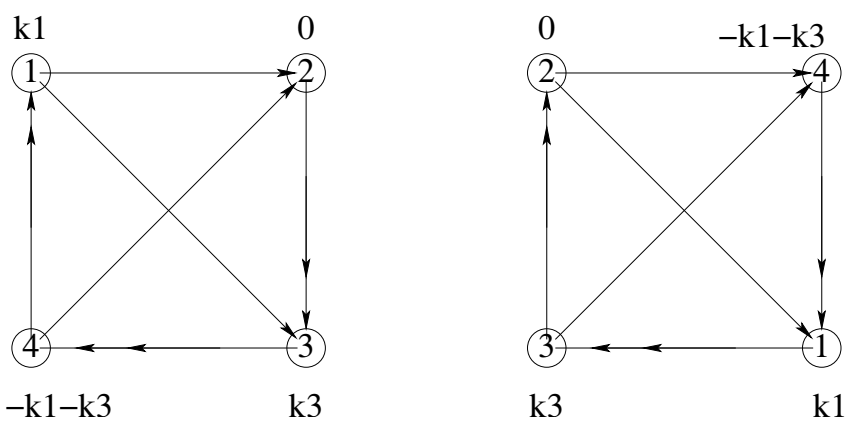

Figure 11. Quiver and CS level for the $\widetilde{d P}_{1\left\{k_{i}\right\}}$ in the two phases related by duality on node 2.

\section{$4.2 \widetilde{d P_{1}}\left\{k_{i}\right\}$}

Here we study the $(2+1) \mathrm{d}$ CS chiral theory whose $(3+1) \mathrm{d}$ parent is $d P_{1}$. In $(3+1) \mathrm{d} d P_{1}$ has only one phase. After Seiberg duality on node 2 the theory has a self similar structure and it is described by the same quiver. The only difference is that we have to change the labels of the groups as $(1 \rightarrow 2,2 \rightarrow 4,3 \rightarrow 1,4 \rightarrow 3)$. The matrix $d, P$ and $Q$ are unchanged by duality. The CS levels in the $C$ matrix change as the labels of the gauge groups do. We give in figure 11 the two phases.

We take the assumption described in the introduction of section 4. Hence we choose the CS level of the group that undergoes duality to be 0 . With this choice the two phases of the $2+1$ dimensional theory (see figure 11) have the same toric diagram.

The superpotential is

$$
W=\varepsilon_{a b} X_{13} X_{34}^{a} X_{41}^{b}+\varepsilon_{a b} X_{42} X_{23}^{a} X_{34}^{b}+\varepsilon_{a b} X_{34} X_{41}^{a} X_{12} X_{23}^{b}
$$

The $d, P, Q$ matrices are

$$
\begin{aligned}
d & =\left(\begin{array}{cccccccccc}
1 & 0 & -1 & 0 & 0 & 0 & -1 & 0 & 0 & 1 \\
0 & 0 & 0 & 0 & -1 & 1 & 0 & 1 & 0 & -1 \\
-1 & 1 & 0 & 1 & 0 & -1 & 0 & -1 & 1 & 0 \\
0 & -1 & 1 & -1 & 1 & 0 & 1 & 0 & -1 & 0
\end{array}\right) \\
P & =\left(\begin{array}{cccccccc}
1 & 1 & 1 & 0 & 0 & 0 & 0 & 0 \\
0 & 0 & 0 & 1 & 1 & 1 & 0 & 0 \\
0 & 0 & 0 & 0 & 0 & 0 & 1 & 1 \\
0 & 0 & 0 & 1 & 1 & 0 & 1 & 0 \\
1 & 1 & 0 & 0 & 0 & 0 & 0 & 1 \\
0 & 0 & 1 & 0 & 0 & 1 & 0 & 0 \\
0 & 0 & 0 & 0 & 0 & 1 & 0 & 1 \\
0 & 0 & 1 & 0 & 0 & 0 & 1 & 0 \\
1 & 0 & 0 & 1 & 0 & 0 & 0 & 0 \\
0 & 1 & 0 & 0 & 1 & 0 & 0 & 0
\end{array}\right)
\end{aligned}
$$



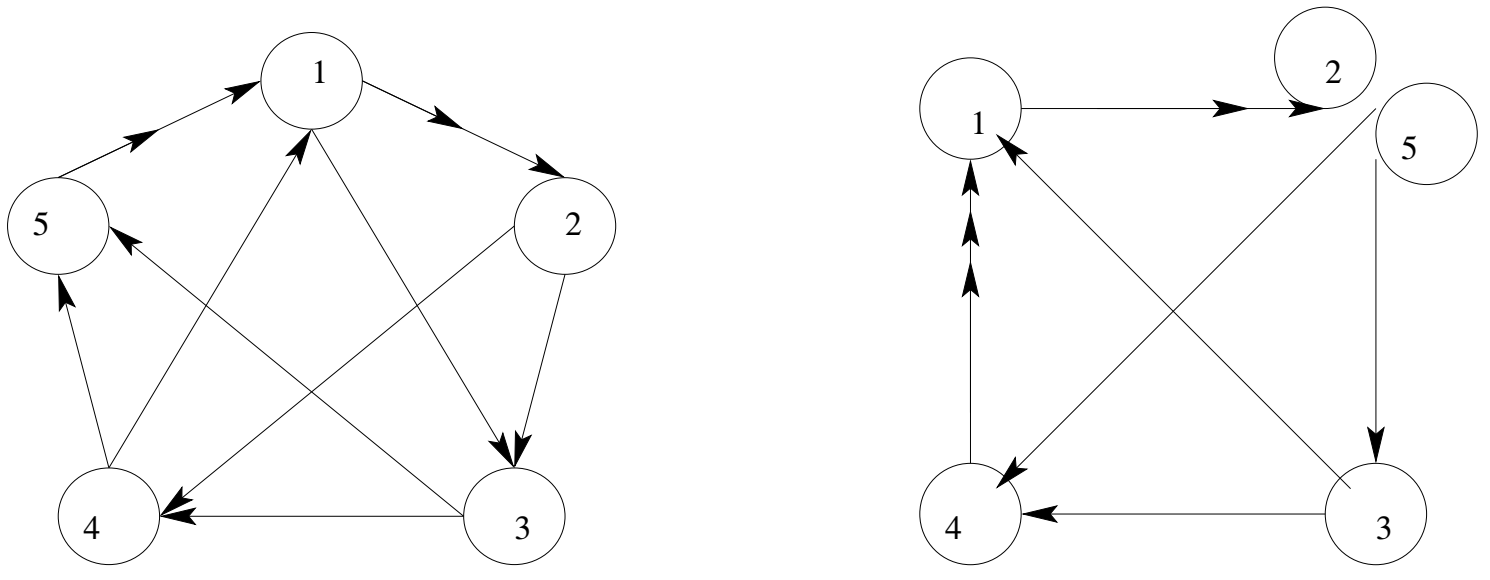

Figure 12. The quivers representing the dual phases of $d P 2$

$$
Q=\left(\begin{array}{cccccccc}
0 & 0 & 1 & 0 & 1 & -1 & -1 & 0 \\
0 & -1 & 1 & 0 & 0 & 0 & 0 & 0 \\
0 & 0 & -1 & 1 & 0 & 0 & 0 & 0 \\
0 & 1 & -1 & -1 & -1 & 1 & 1 & 0
\end{array}\right)
$$

Following the relabeling of the gauge groups, the $C$ matrix in the two phases are

$$
C_{1}=\left(\begin{array}{cccc}
1 & 1 & 1 & 1 \\
k_{1} & 0 & k_{3} & -k_{1}-k_{3}
\end{array}\right) \quad C_{2}=\left(\begin{array}{cccc}
1 & 1 & 1 & 1 \\
0 & -k_{1}-k_{3} & k_{1} & k_{3}
\end{array}\right)
$$

The toric diagram for the first theory is given by the matrix $G_{t}^{(1)}$

$$
G_{t}^{(1)}=\left(\begin{array}{cccccccc}
1 & 1 & 1 & 1 & 1 & 1 & 1 & 1 \\
0 & 0 & 0 & 0 & 0 & -1 & 1 & 0 \\
-1 & 0 & 0 & 0 & 1 & 1 & 0 & 0 \\
k_{1}+k_{3} & k_{1} & k_{1} & k_{1}+k_{3} & k_{1} & k_{1} & 0 & 0
\end{array}\right)
$$

The toric diagram of the dual theory, up to $\mathrm{SL}(4, Z)$ transformation, is

$$
G_{t}^{(2)}=\left(\begin{array}{cccccccc}
1 & 1 & 1 & 1 & 1 & 1 & 1 & 1 \\
0 & 0 & 0 & 0 & 0 & -1 & 1 & 0 \\
-1 & 0 & 0 & 0 & 1 & 1 & 0 & 0 \\
k_{1}+k_{3} & k_{1}+k_{3} & 0 & k_{1} & k_{1} & 0 & k_{1} & k_{1}+k_{3}
\end{array}\right)
$$

This shows that the two systems of vectors give the same toric diagram and that the two theories have the same abelian moduli space also in the $2+1$ dimensions, provided $k_{2}=0$.

\section{$4.3 \widetilde{d P_{2}}\left\{k_{i}\right\}$}

We analyze the $(2+1)$ d CS chiral theory with $d P_{2}$ as $(3+1)$ d parents. The $4 D$ theory has two inequivalent phases. The two phases are connected by duality on node 5 and are reported in figure 12 . 
The constraint on the CS levels explained in the introduction of this section imposes $k_{2}=0$ and $k_{5}=0$. Under this assumption the two phases have the same toric diagram also for the $(2+1)$ d CS theory.

The superpotential for the two phases are

$$
\begin{aligned}
W_{I}= & X_{13} X_{34} X_{41}-Y_{12} X_{24} X_{41}+X_{12} X_{24} X_{45} Y_{51}-X_{13} X_{35} Y_{51} \\
& +Y_{12} X_{23} X_{35} X_{51}-X_{12} X_{23} X_{34} X_{45} X_{51} \\
W_{I I}= & Y_{41} X_{15} X_{54}-X_{31} X_{15} X_{53}+Y_{12} X_{23} X_{31}-Y_{12} X_{24} X_{41}+Y_{15} X_{53} X_{34} X_{41} \\
& -Z_{41} Y_{15} X_{54}+X_{12} X_{24} Z_{41}-X_{12} X_{23} X_{34} Y_{41}
\end{aligned}
$$

We compute the toric diagrams $G_{t}^{(I)}$ and $G_{t}^{(I I)}$ for the two phases

$$
\begin{aligned}
G_{t}^{(I)} & =\left(\begin{array}{cccccccccc}
1 & 1 & 1 & 1 & 1 & 1 & 1 & 1 & 1 & 1 \\
0 & 0 & 1 & 0 & 0 & 0 & 0 & -1 & 0 & -1 \\
0 & -1 & 0 & 0 & 1 & 0 & 0 & 0 & 0 & -1 \\
k_{1} & k_{1} & k_{1} & k_{1} & 0 & 0 & 0 & 0 & -k_{3} & -k_{3}
\end{array}\right) \\
G_{t}^{(I I)} & =\left(\begin{array}{cccccccccccc}
1 & 1 & 1 & 1 & 1 & 1 & 1 & 1 & 1 & 1 & 1 \\
0 & -1 & 0 & 0 & 1 & 0 & 0 & 0 & 0 & 0 & -1 \\
0 & -1 & -1 & 0 & 0 & 0 & 0 & 1 & 0 & 0 & 0 \\
0 & -k_{3} & k_{1} & 0 & k_{1} & -k_{3} & k_{1} & 0 & 0 & 0 & 0
\end{array}\right)
\end{aligned}
$$

They result the same.

\section{$4.4 \widetilde{d P_{3}}\left\{k_{i}\right\}$}

Here we study $\widetilde{d P}_{3}\left\{k_{i}\right\}$. This theory has four phases in four dimensions, with superpotentials

$$
\begin{aligned}
W_{I}= & X_{13} X_{34} X_{46} X_{61}-X_{24} X_{46} X_{62}+X_{12} X_{24} X_{45} X_{51}-X_{13} X_{35} X_{51} \\
& +X_{23} X_{35} X_{56} X_{62}-X_{12} X_{23} X_{34} X_{45} X_{56} X_{61} \\
W_{I I}= & X_{13} X_{34} X_{41}-X_{13} X_{35} X_{51}+X_{23} X_{35} X_{52}-X_{26} X_{65} X_{52}+X_{16} X_{65} Y_{51} \\
& -X_{16} X_{64} X_{41}+X_{12} X_{26} X_{64} X_{45} X_{51}-X_{12} X_{23} X_{34} X_{45} Y_{51} \\
W_{I I I}= & X_{23} X_{35} X_{52}-X_{26} X_{65} X_{52}+X_{14} X_{46} X_{65} Y_{51}-X_{12} X_{23} Y_{35} Y_{51}+X_{43} Y_{35} X_{54} \\
& -Y_{65} X_{54} X_{46}+X_{12} X_{26} Y_{65} X_{51}-X_{14} X_{43} X_{35} X_{51} \\
W_{I V}= & X_{23} X_{35} X_{52}-X_{52} X_{26} X_{65}+X_{65} Z_{54} X_{46}-Z_{54} X_{41} Y_{15}+Y_{15} Z_{52} X_{21}-Z_{52} X_{23} Y_{35} \\
& +Y_{35} X_{54} X_{43}-X_{54} X_{46} Y_{65}+Y_{65} Y_{52} X_{26}-Y_{52} X_{21} X_{15}+X_{15} Y_{54} X_{41}-Y_{54} X_{43} X_{35}
\end{aligned}
$$

Phases (II, III, IV) are computed from phases (I, II, III) by dualizing nodes $(6,4,1)$ respectively. The quivers associated with each phase are given in figure 13 . We now show the equivalence of phases (I,II), (II,III) and (III,IV) by choosing $\left(k_{3}=k_{6}=0\right.$ ), 

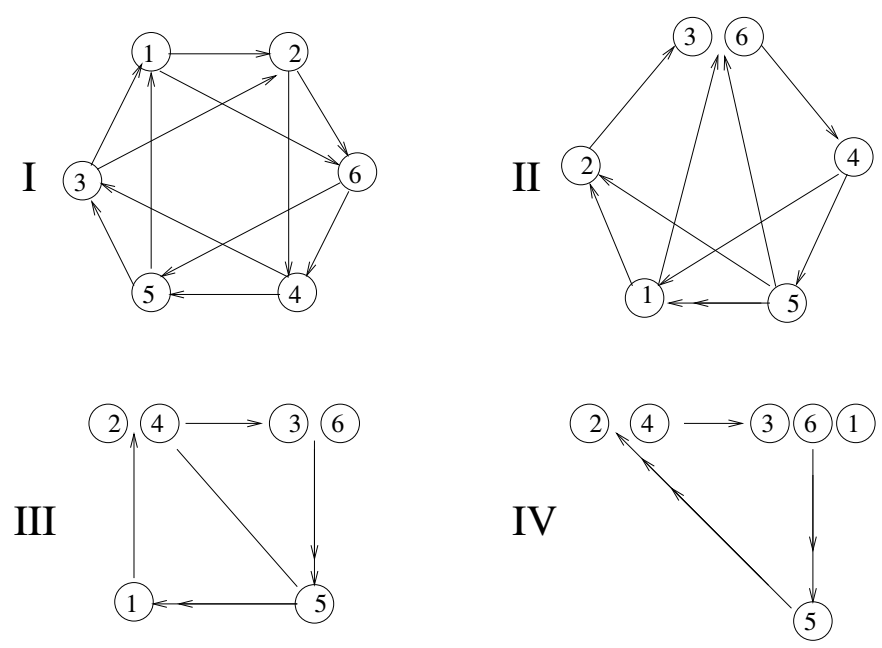

Figure 13. The quiver of $d P_{3}$

$\left(k_{2}=k_{4}=0\right)$ and $\left(k_{1}=k_{3}=k_{6}=0\right)$ respectively. For phases $(I, I I)$ we have

$$
\begin{aligned}
G_{t}^{(I)} & =\left(\begin{array}{cccccccccc}
1 & 1 & 1 & 1 & 1 & 1 & 1 & 1 & 1 & 1 \\
-1 & 0 & 0 & 0 & 0 & 1 & 0 & 0 & 0 & -1 \\
-1 & 0 & -1 & 0 & 0 & 1 & 0 & 1 & 0 & 0 \\
k_{1}+k_{2} & 0 & k_{1} & k_{1}+k_{2}+k_{4} & k_{1}+k_{2} & 0 & k_{1} & k_{1}+k_{2}+k_{4} & k_{1}+k_{2} & k_{1}+k_{2} \\
1 & 1 \\
1 & 0 \\
0 & 0 \\
0 & 0
\end{array}\right) \\
G_{t}^{(I I)} & =\left(\begin{array}{ccccccccc}
1 &
\end{array}\right. \\
& \\
0 &
\end{aligned}
$$

For phases $(I I, I I I)$ we have

$$
G_{t}^{(I I)}=\left(\begin{array}{ccccccccc}
1 & 1 & 1 & 1 & 1 & 1 & 1 & 1 & 1 \\
0 & 1 & 1 & 0 & 0 & -1 & 0 & 0 & 0 \\
0 & 0 & 1 & 0 & 0 & 0 & 0 & 1 & 0 \\
-k_{1} & k_{3}+k_{5} & k_{3}+k_{5} & k_{3}+k_{5} & -k_{1} & -k_{1}-k_{3} & k_{5} & -k_{1}-k_{3} & k_{5}
\end{array}\right.
$$




$$
\begin{aligned}
& \left.\begin{array}{lccc}
1 & 1 & 1 & 1 \\
0 & 0 & -1 & 0 \\
0 & 0 & -1 & -1 \\
k_{1}-k_{3} & 0 & 0 & 0
\end{array}\right) \\
& G_{t}^{(I I I)}=\left(\begin{array}{ccccccccccc}
1 & 1 & 1 & 1 & 1 & 1 & 1 & 1 & 1 & 1 & 1 \\
0 & 1 & 0 & 0 & 1 & 0 & -1 & 0 & 0 & -1 & 0 \\
0 & 1 & -1 & 0 & 0 & 0 & -1 & 0 & 0 & 0 & 0 \\
0 & k_{3}+k_{5} & 0 & -k_{1} & k_{3}+k_{5} & -k_{1} & 0 & k_{3}+k_{5} & k_{5} & -k_{1}-k_{3} & -k_{1}
\end{array}\right. \\
& \left.\begin{array}{ccc}
1 & 1 & 1 \\
0 & 0 & 0 \\
0 & 1 & 0 \\
-k_{1} & -k_{1}-k_{3} & -k_{1}-k_{3}
\end{array}\right)
\end{aligned}
$$

For phases $(I I I, I V)$ we have

$$
\begin{aligned}
G_{t}^{(I I I)} & =\left(\begin{array}{cccccccccccccc}
1 & 1 & 1 & 1 & 1 & 1 & 1 & 1 & 1 & 1 & 1 & 1 & 1 & 1 \\
0 & -1 & 1 & 0 & 0 & 0 & 1 & 0 & 0 & 0 & 0 & 0 & -1 & 0 \\
0 & -1 & 0 & 0 & -1 & 0 & 1 & 0 & 0 & 1 & 0 & 0 & 0 & 0 \\
k_{2}+k_{4} & k_{2} & k_{2}+k_{4} & k_{2} & k_{2}+k_{4} & k_{2}+k_{4} & k_{4} & 0 & 0 & 0 & 0 & k_{4} & 0 & 0
\end{array}\right) \\
G_{t}^{(I V)} & =\left(\begin{array}{ccccccccccc}
1 & 1 & & 1 & 1 & 1 & 1 & & 1 & 1 & 1 \\
0 & 0 & & 0 & 0 & 1 & 0 & 0 & 0 & 0 \\
0 & 0 & & 0 & 0 & 0 & -1 & 0 & 0 & 0 \\
k_{2}+k_{4} & k_{2}+k_{4} & k_{2}+k_{4} & k_{2}+k_{4} & k_{2}+k_{4} & k_{2}+k_{4} & k_{2}+k_{4} & k_{2}+k_{4} & k_{2}+k_{4} \\
1 & 1 & 1 & 1 & 1 & 1 & 1 & 1 \\
0 & 0 & 1 & 0 & -1 & 0 & 0 & -1 \\
0 & 0 & 1 & 0 & -1 & 1 & 0 & 0 \\
k_{2}+k_{4} & k_{4} & k_{4} & k_{2} & k_{2} & 0 & 0 & 0
\end{array}\right)
\end{aligned}
$$

\section{$4.5 \widetilde{Y^{32}}\left\{k_{i}\right\}$}

This is the last chiral theory we analyze. In this case, after duality, there is not an identification between the gauge group that undergoes duality with other groups. This implies that the assumptions of section 4 impose only $k_{g}=0$, where $k_{g}$ is the CS level of the dualized gauge group. As for the case of $d P_{1}$ and all the $Y^{p, p-1}$ theories, the $Y^{3,2}$ theory is self similar under four dimensional Seiberg duality. We can evaluate $\mathcal{M}_{4}$ for one phase and then the toric diagram associated with a dual phase is given by an appropriate change of the $D$-term modding matrix.

We fix the conventions on the groups by giving the tiling of the two dual phases, see figure 14 . The two phases are connected by duality on node 5 , so we set $k_{5}=0$. 

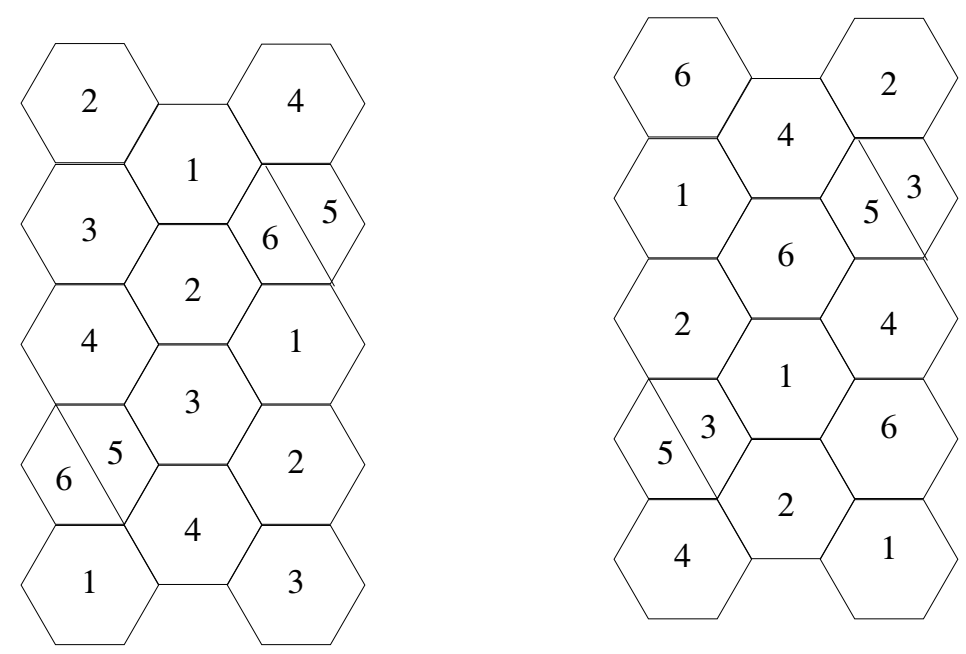

Figure 14. The tiling for the two dual phases of $Y^{32}$. Seiberg duality has been performed on groups 5 .

The CS matrices for the two dual phases are:

$$
\begin{aligned}
C_{1} & =\left(\begin{array}{ccccccccc}
1 & 1 & 1 & 1 & 1 & \multicolumn{4}{c}{1} \\
k_{1} & k_{2} & k_{3} & k_{4} & 0 & -k_{1}-k_{2}-k_{3}-k_{4}
\end{array}\right) \\
C_{2} & =\left(\begin{array}{cccccccc}
1 & \multicolumn{1}{c}{1} & 1 & 1 & 1 & 1 \\
k_{4} & -k_{1}-k_{2}-k_{3}-k_{4} & k_{1} & k_{2} & k_{3} & 0
\end{array}\right)
\end{aligned}
$$

The toric diagrams are encoded in the $G_{t}$ matrices.

$$
\begin{aligned}
& G_{t}^{(1)}=\left(\begin{array}{cccccccccc}
1 & 1 & 1 & 1 & 1 & 1 & 1 & 1 & 1 & 1 \\
0 & 0 & 0 & 0 & 0 & 0 & 0 & 0 & 0 & 0 \\
2 & 1 & 1 & 1 & 0 & 1 & 0 & 0 & 1 & 0 \\
k_{3}+k_{4} & 0 & -k_{6} & k_{2}+k_{3}+k_{4} & k_{2} & k_{3}+k_{4} & 0 & -k_{6} & k_{4} & -k_{3}
\end{array}\right. \\
& \left.\begin{array}{cccccccc}
1 & 1 & 1 & 1 & 1 & 1 & 1 & 1 \\
0 & 0 & 1 & 0 & 0 & 0 & 0 & -1 \\
0 & 0 & -1 & -1 & 1 & 0 & 0 & 0 \\
k_{1}+k_{2}+k_{4} & k_{2}+k_{4} & k_{1}+2 k_{2}+k_{4} & k_{2}-k_{3} & 0 & k_{2} & 0 & 0
\end{array}\right) \\
& G_{t}^{(2)}=\left(\begin{array}{ccccccccc}
1 & 1 & 1 & 1 & 1 & 1 & 1 & 1 & 1 \\
0 & 0 & 0 & 0 & 0 & 0 & 0 & 0 & 0 \\
2 & 1 & 1 & 1 & 0 & 1 & 0 & 0 & 1 \\
k_{3}+k_{4} & k_{4} & k_{4} & 0 & -k_{3} & -k_{6} & k_{1}+k_{2}+k_{4} & k_{1}+k_{2}+k_{3} & k_{2}+k_{3}+k_{4}
\end{array}\right. \\
& \left.\begin{array}{ccccccccc}
1 & 1 & 1 & 1 & 1 & 1 & 1 & 1 & 1 \\
0 & 0 & 0 & 1 & 0 & 0 & 0 & 0 & -1 \\
0 & 0 & 0 & -1 & -1 & 1 & 0 & 0 & 0 \\
k_{2}+k_{4} & k_{2}+k_{4} & k_{2} & k_{1}+2 k_{2}+k_{4} & k_{2}-k_{3} & k_{3}+k_{4} & 0 & -k_{6} & 0
\end{array}\right)
\end{aligned}
$$

and they coincide for arbitrary $k_{1}, k_{2}, k_{3}, k_{4}$, remind $k_{6}=-k_{1}-k_{2}-k_{3}-k_{4}$. 


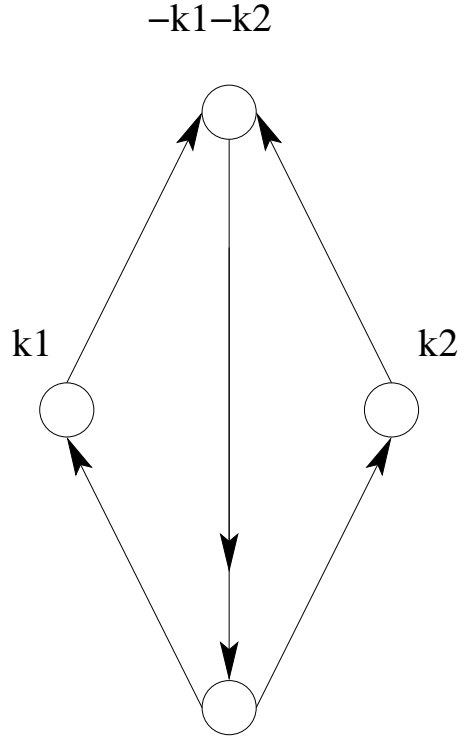

0

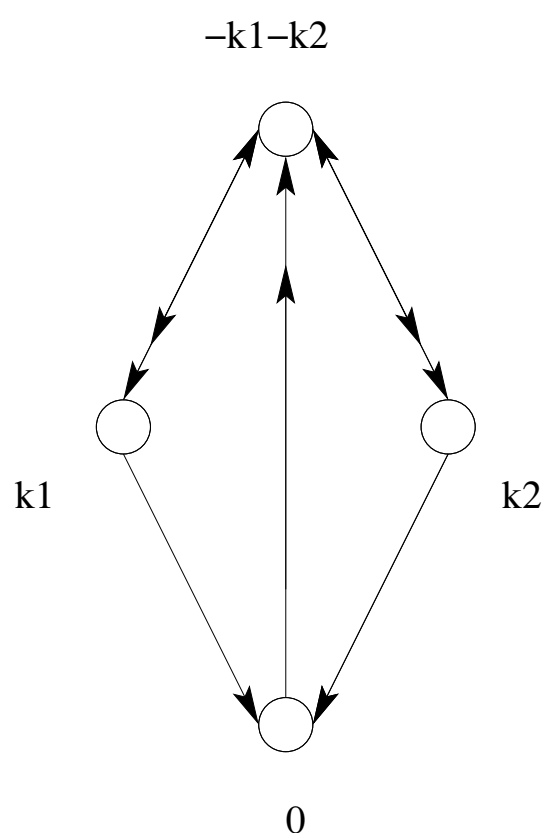

k2

Figure 15. The quiver for $Q^{111}$ in the two dual phases.

\section{Dualities for CS theories without $4 \mathrm{~d}$ parents}

Three dimensional CS theories with four dimensional parents are a subset of all the possible 3d CS theories [29]. For CS theories without four dimensional parents we miss in principle the intuition from the $4 \mathrm{~d}$ Seiberg duality. In this short section we see that we can still describe a subset of $3 \mathrm{~d}$ CS theories with the same mesonic moduli space if we just apply the rules we learned in the previous sections.

We study a case associated with $Q^{111}$. We show that by performing a Seiberg-like duality and by setting the CS level of the dualized gauge group to zero, the toric diagrams of the two models coincide.

\subsection{Example}

The theory is described by the quiver given in figure 15a. It is a generalization of the $C\left(Q_{1,1,1}\right)$ with arbitrary CS levels [29]. The superpotential is

$$
W=X_{41} X_{13} X_{34}^{1} X_{42} X_{23} X_{34}^{2}-X_{41} X_{13} X_{34}^{2} X_{42} X_{23} X_{34}^{1}
$$

The toric diagram for $k_{4}=0$ is given by

$$
G_{t}=\left(\begin{array}{cccccc}
1 & 1 & 1 & 1 & 1 & 1 \\
1 & 0 & 1 & 0 & 1 & 0 \\
1 & 0 & 0 & 1 & 1 & 0 \\
k_{1} & k_{2} & 0 & 0 & 0 & 0
\end{array}\right)
$$


Seiberg duality on node $N_{4}$ gives the superpotential

$$
\begin{aligned}
W= & X_{13} X_{32}^{(1)} X_{23} X_{31}^{(2)}-X_{13} X_{32}^{(2)} X_{23} X_{31}^{(1)}+X_{24} X_{43}^{(2)} X_{32}^{(2)}-X_{24} X_{43}^{(1)} X_{32}^{(1)} \\
& +X_{14} X_{43}^{(1)} X_{31}^{(1)}-X_{14} X_{43}^{(2)} X_{31}^{(2)}
\end{aligned}
$$

and the theory is described by the quiver given in figure 15b. The toric diagram in this case is

$$
G_{t}=\left(\begin{array}{cccccccc}
1 & 1 & 1 & 1 & 1 & 1 & 1 & 1 \\
1 & 0 & 1 & 0 & 1 & 0 & 1 & 0 \\
1 & 0 & 1 & 0 & 1 & 1 & 0 & 0 \\
k_{1} & k_{2} & k_{1} & k_{2} & 0 & 0 & 0 & 0
\end{array}\right)
$$

and it is equivalent to the toric diagram for the first phase.

\section{Conclusion}

In this paper we have some advances towards the understanding of toric duality for M2 branes. Generalizing the work of [34, 35], we proposed a Seiberg-like duality for non-chiral three dimensional CS matter theories and we verified that the mesonic moduli space of dual theories is indeed the same four-fold Calabi Yau probed by the M2 branes. In the chiral case and in the case in which the three dimensional theories do not have a four dimensional parent, the situation is more complicated. However, fixing to zero the value of some of the Chern-Simons levels, we were able to realize toric dual pairs.

We have just analyzed the mesonic moduli space, it would be important to study the complete moduli space, including baryonic operators.

For the non-chiral case the two main limitations are the lack of understanding of the transformation rule for the rank of the gauge groups and the fact that we forced to zero some of the $k_{i}$. It is reasonable that there exist some more general and precise transformation rules and we would like to investigate them.

We concentrated on Seiberg-like transformations, but it is well known that in three dimension there exist duality maps that change the number of gauge group factors. It would be interesting to systematically study these more general transformations.

A lot of possible directions and generalizations are opening up and after these first steps we hope to step up.

\section{Acknowledgments}

We are happy to thank Alberto Zaffaroni for many nice discussions and Ami Hanany for comments. We are grateful to the authors of $[59,60]$ for informing us about their research on similar topics.

A. A. and L. G. are supported in part by INFN, in part by MIUR under contract 2007-5ATT78-002 and in part by the European Commission RTN programme MRTN-CT2004-005104. D. F. is supported by CNRS and ENS Paris. A. M. is supported in part by the Belgian Federal Science Policy Office through the Interuniversity Attraction Pole IAP VI/11, by the European Commission FP6 RTN programme MRTN-CT-2004-005104 and by FWO-Vlaanderen through project G.0428.06. 


\section{A Parity anomaly}

We briefly review parity anomaly for $3 \mathrm{D}$ gauge theories [55-57] and the parity anomaly matching argument [36]. In three dimensions there are no local gauge anomalies. However gauge invariance can require the introduction of a classical Chern-Simons term, which breaks parity. This is referred to as parity anomaly.

For abelian theories with multiple U(1)'s, there is a parity anomaly if

$$
\mathcal{A}_{i j}=\frac{1}{2} \sum_{\text {fermion }}\left(q_{f}\right)_{i}\left(q_{f}\right)_{j} \in \mathbb{Z}+\frac{1}{2}
$$

Here $\left(q_{f}\right)_{i}$ is the charge of the fermion $f$ under the $\mathrm{U}(1)_{i}$. We work in a basis where all the charges are integers.

Parity anomaly matching. In the context of dualities in 4D gauge theory, a relevant tool has been the 't Hooft anomaly matching between the electric and the magnetic description. Having some global symmetries, we suppose that they are gauged and we compute their anomaly. The result of this computation should be equal in the two dual descriptions. The same technique can be used here for the parity anomaly. We suppose we gauge the global U(1)'s of the theory, and we compute their parity anomaly both in the electric and in the magnetic description. The two computations should match.

The parity anomaly matching is much weaker then the 't Hooft one. Indeed in 4D the precise anomalies associated with gauging global symmetries must match. In 3D there is a weaker $\mathbb{Z}_{2}$ type condition.

For the $\widehat{L}_{\left\{k_{i}\right\}}$ theories, parity anomaly matching is obeyed by the Seiberg like duality we propose.

In chiral theories, parity anomaly matching can be non trivial between dual phases if fractional branes are introduced by the duality.

As an example we analyze the toric chiral $(2+1)$ d CS theory which has as $(3+1)$ d parents $d P_{2}$. We consider the parity anomaly associated to the two $\mathrm{U}(1)$ flavour symmetries $F_{1}$ and $F_{2}$. The charges of the chiral fields of the theory under these symmetries can be derived from [41]. The electric theory has equal ranks $n$. In the magnetic description we set rank $n+k$ for the dualized gauge group (number 5 ) and $n$ for the others. The integer $k$ counts the number of fractional branes introduced in the duality. The parity anomaly matrices are

$$
\mathcal{A}_{\text {ele }} \in\left(\begin{array}{l}
\mathbb{Z} \mathbb{Z} \\
\mathbb{Z} \mathbb{Z}
\end{array}\right)
$$

and

$$
\mathcal{A}_{\text {mag }} \in\left(\begin{array}{cc}
\mathbb{Z} & \mathbb{Z}+\frac{n k}{2} \\
\mathbb{Z}+\frac{n k}{2} & \mathbb{Z}
\end{array}\right)
$$

One can see that in the electric description there are no parity anomalies. In the magnetic description the off diagonal components of $\mathcal{A}_{\text {mag }}$ can instead lead to parity anomaly if $k n$ is odd. If we set $k=0$ the electric and magnetic theories satisfies the parity anomaly matching for the two flavour symmetries. 
Open Access. This article is distributed under the terms of the Creative Commons Attribution Noncommercial License which permits any noncommercial use, distribution, and reproduction in any medium, provided the original author(s) and source are credited.

\section{References}

[1] B. Feng, A. Hanany and Y.-H. He, D-brane gauge theories from toric singularities and toric duality, Nucl. Phys. B 595 (2001) 165 [hep-th/0003085] [SPIRES].

[2] B. Feng, A. Hanany and Y.-H. He, Phase structure of D-brane gauge theories and toric duality, JHEP 08 (2001) 040 [hep-th/0104259] [SPIRES].

[3] B. Feng, S. Franco, A. Hanany and Y.-H. He, Symmetries of toric duality, JHEP 12 (2002) 076 [hep-th/0205144] [SPIRES].

[4] C.E. Beasley and M.R. Plesser, Toric duality is Seiberg duality, JHEP 12 (2001) 001 [hep-th/0109053] [SPIRES].

[5] B. Feng, A. Hanany, Y.-H. He and A.M. Uranga, Toric duality as Seiberg duality and brane diamonds, JHEP 12 (2001) 035 [hep-th/0109063] [SPIRES].

[6] J.H. Schwarz, Superconformal Chern-Simons theories, JHEP 11 (2004) 078 [hep-th/0411077] [SPIRES].

[7] J. Bagger and N. Lambert, Modeling multiple M2's, Phys. Rev. D 75 (2007) 045020 [hep-th/0611108] [SPIRES].

[8] J. Bagger and N. Lambert, Gauge symmetry and supersymmetry of multiple M2-branes, Phys. Rev. D 77 (2008) 065008 [arXiv:0711.0955] [SPIRES].

[9] J. Bagger and N. Lambert, Comments on multiple M2-branes, JHEP 02 (2008) 105 [arXiv:0712.3738] [SPIRES].

[10] A. Gustavsson, Algebraic structures on parallel M2-branes, Nucl. Phys. B 811 (2009) 66 [arXiv: 0709.1260] [SPIRES].

[11] A. Gustavsson, Selfdual strings and loop space Nahm equations, JHEP 04 (2008) 083 [arXiv:0802.3456] [SPIRES].

[12] M. Van Raamsdonk, Comments on the Bagger-Lambert theory and multiple M2-branes, JHEP 05 (2008) 105 [arXiv: 0803.3803] [SPIRES].

[13] S. Mukhi and C. Papageorgakis, M2 to D2, JHEP 05 (2008) 085 [arXiv:0803.3218] [SPIRES].

[14] M. Fujita, W. Li, S. Ryu and T. Takayanagi, Fractional quantum Hall effect via holography: Chern-Simons, edge states and hierarchy, JHEP 06 (2009) 066 [arXiv:0901.0924] [SPIRES].

[15] O. Aharony, O. Bergman, D.L. Jafferis and J. Maldacena, $\mathcal{N}=6$ superconformal Chern-Simons-matter theories, M2-branes and their gravity duals, JHEP 10 (2008) 091 [arXiv:0806.1218] [SPIRES].

[16] M. Benna, I. Klebanov, T. Klose and M. Smedback, Superconformal Chern-Simons theories and $\mathrm{AdS}_{4} / \mathrm{CFT}_{3}$ correspondence, JHEP 09 (2008) 072 [arXiv:0806.1519] [SPIRES]. 
[17] K. Hosomichi, K.-M. Lee, S. Lee, S. Lee and J. Park, $\mathcal{N}=4$ superconformal Chern-Simons theories with hyper and twisted hyper multiplets, JHEP 07 (2008) 091 [arXiv:0805.3662] [SPIRES].

[18] K. Hosomichi, K.-M. Lee, S. Lee, S. Lee and J. Park, $\mathcal{N}=5,6$ superconformal Chern-Simons theories and M2-branes on orbifolds, JHEP 09 (2008) 002 [arXiv:0806.4977] [SPIRES].

[19] M. Schnabl and Y. Tachikawa, Classification of $\mathcal{N}=6$ superconformal theories of ABJM type, arXiv:0807.1102 [SPIRES].

[20] D. Martelli and J. Sparks, Moduli spaces of Chern-Simons quiver gauge theories and $A d S_{4} / C F T_{3}$, Phys. Rev. D 78 (2008) 126005 [arXiv:0808.0912] [SPIRES].

[21] A. Hanany and A. Zaffaroni, Tilings, Chern-Simons theories and M2 branes, JHEP 10 (2008) 111 [arXiv:0808.1244] [SPIRES].

[22] A. Hanany, D. Vegh and A. Zaffaroni, Brane tilings and M2 branes, JHEP 03 (2009) 012 [arXiv:0809.1440] [SPIRES].

[23] D.L. Jafferis and A. Tomasiello, A simple class of $\mathcal{N}=3$ gauge/gravity duals, JHEP 10 (2008) 101 [arXiv:0808.0864] [SPIRES].

[24] D. Gaiotto and A. Tomasiello, The gauge dual of Romans mass, JHEP 01 (2010) 015 [arXiv: 0901.0969] [SPIRES].

[25] Y. Imamura and K. Kimura, On the moduli space of elliptic Maxwell-Chern-Simons theories, Prog. Theor. Phys. 120 (2008) 509 [arXiv:0806.3727] [SPIRES].

[26] Y. Imamura and K. Kimura, $\mathcal{N}=4$ Chern-Simons theories with auxiliary vector multiplets, JHEP 10 (2008) 040 [arXiv:0807.2144] [SPIRES].

[27] Y. Imamura and S. Yokoyama, $\mathcal{N}=4$ Chern-Simons theories and wrapped M5-branes in their gravity duals, Prog. Theor. Phys. 121 (2009) 915 [arXiv:0812.1331] [SPIRES].

[28] A. Hanany and Y.-H. He, M2-branes and quiver Chern-Simons: a taxonomic study, arXiv:0811. 4044 [SPIRES].

[29] S. Franco, A. Hanany, J. Park and D. Rodriguez-Gomez, Towards M2-brane theories for generic toric singularities, JHEP 12 (2008) 110 [arXiv:0809.3237] [SPIRES].

[30] K. Ueda and M. Yamazaki, Toric Calabi-Yau four-folds dual to Chern-Simons-matter theories, JHEP 12 (2008) 045 [arXiv:0808.3768] [SPIRES].

[31] Y. Imamura and K. Kimura, Quiver Chern-Simons theories and crystals, JHEP 10 (2008) 114 [arXiv:0808.4155] [SPIRES].

[32] A. Kapustin and M.J. Strassler, On mirror symmetry in three dimensional Abelian gauge theories, JHEP 04 (1999) 021 [hep-th/9902033] [SPIRES].

[33] K.A. Intriligator and N. Seiberg, Mirror symmetry in three dimensional gauge theories, Phys. Lett. B 387 (1996) 513 [hep-th/9607207] [SPIRES].

[34] O. Aharony, O. Bergman and D.L. Jafferis, Fractional M2-branes, JHEP 11 (2008) 043 [arXiv:0807.4924] [SPIRES].

[35] A. Giveon and D. Kutasov, Seiberg duality in Chern-Simons theory, Nucl. Phys. B 812 (2009) 1 [arXiv:0808.0360] [SPIRES].

[36] O. Aharony, A. Hanany, K.A. Intriligator, N. Seiberg and M.J. Strassler, Aspects of $\mathcal{N}=2$ supersymmetric gauge theories in three dimensions, Nucl. Phys. B 499 (1997) 67 [hep-th/9703110] [SPIRES]. 
[37] A. Karch, Seiberg duality in three dimensions, Phys. Lett. B 405 (1997) 79 [hep-th/9703172] [SPIRES].

[38] O. Aharony, IR duality in $D=3 \mathcal{N}=2$ supersymmetric $\mathrm{USp}\left(2 N_{c}\right)$ and $\mathrm{U}\left(N_{c}\right)$ gauge theories, Phys. Lett. B 404 (1997) 71 [hep-th/9703215] [SPIRES].

[39] D. Forcella, A. Hanany, Y.-H. He and A. Zaffaroni, The master space of $\mathcal{N}=1$ gauge theories, JHEP 08 (2008) 012 [arXiv:0801.1585] [SPIRES].

[40] D. Forcella, A. Hanany, Y.-H. He and A. Zaffaroni, Mastering the master space, Lett. Math. Phys. 85 (2008) 163 [arXiv:0801.3477] [SPIRES].

[41] D. Forcella, A. Hanany and A. Zaffaroni, Master space, Hilbert series and Seiberg duality, JHEP 07 (2009) 018 [arXiv:0810.4519] [SPIRES].

[42] S. Benvenuti and M. Kruczenski, From Sasaki-Einstein spaces to quivers via BPS geodesics: $L^{p, q \mid r}, J H E P 04$ (2006) 033 [hep-th/0505206] [SPIRES].

[43] A. Butti, D. Forcella and A. Zaffaroni, The dual superconformal theory for $L^{p, q \mid r}$ manifolds, JHEP 09 (2005) 018 [hep-th/0505220] [SPIRES].

[44] S. Franco et al., Gauge theories from toric geometry and brane tilings, JHEP 01 (2006) 128 [hep-th/0505211] [SPIRES].

[45] A. Hanany and E. Witten, Type IIB superstrings, BPS monopoles and three-dimensional gauge dynamics, Nucl. Phys. B 492 (1997) 152 [hep-th/9611230] [SPIRES].

[46] T. Kitao, K. Ohta and N. Ohta, Three-dimensional gauge dynamics from brane configurations with (p,q)-fivebrane, Nucl. Phys. B 539 (1999) 79 [hep-th/9808111] [SPIRES].

[47] O. Bergman, A. Hanany, A. Karch and B. Kol, Branes and supersymmetry breaking in $3 D$ gauge theories, JHEP 10 (1999) 036 [hep-th/9908075] [SPIRES].

[48] A.M. Uranga, Brane configurations for branes at conifolds, JHEP 01 (1999) 022 [hep-th/9811004] [SPIRES].

[49] S. Elitzur, A. Giveon and D. Kutasov, Branes and $\mathcal{N}=1$ duality in string theory, Phys. Lett. B 400 (1997) 269 [hep-th/9702014] [SPIRES].

[50] V. Niarchos, Seiberg duality in Chern-Simons theories with fundamental and adjoint matter, JHEP 11 (2008) 001 [arXiv:0808.2771] [SPIRES].

[51] E. Witten, Supersymmetric index of three-dimensional gauge theory, hep-th/9903005 [SPIRES].

[52] K. Ohta, Supersymmetric index and s-rule for type IIB branes, JHEP 10 (1999) 006 [hep-th/9908120] [SPIRES].

[53] A. Hanany and K.D. Kennaway, Dimer models and toric diagrams, hep-th/0503149 [SPIRES].

[54] S. Franco, A. Hanany, K.D. Kennaway, D. Vegh and B. Wecht, Brane dimers and quiver gauge theories, JHEP 01 (2006) 096 [hep-th/0504110] [SPIRES].

[55] A.J. Niemi and G.W. Semenoff, Axial anomaly induced fermion fractionization and effective gauge theory actions in odd dimensional space-times, Phys. Rev. Lett. 51 (1983) 2077 [SPIRES].

[56] L. Álvarez-Gaumé and E. Witten, Gravitational anomalies, Nucl. Phys. B 234 (1984) 269 [SPIRES]. 
[57] A.N. Redlich, Parity violation and gauge noninvariance of the effective gauge field action in three-dimensions, Phys. Rev. D 29 (1984) 2366 [SPIRES].

[58] S. Kim, S. Lee, S. Lee and J. Park, Abelian gauge theory on M2-brane and toric duality, Nucl. Phys. B 797 (2008) 340 [arXiv:0705.3540] [SPIRES].

[59] S. Franco, I.R. Klebanov and D. Rodriguez-Gomez, M2-branes on orbifolds of the cone over $Q^{1,1,1}$, JHEP 08 (2009) 033 [arXiv:0903.3231] [SPIRES].

[60] J. Davey, A. Hanany, N. Mekareeya and G. Torri, Phases of M2-brane theories, JHEP 06 (2009) 025 [arXiv:0903.3234] [SPIRES]. 